\title{
Methylome-based cell-of-origin modeling (Methyl-COOM) identifies aberrant expression of immune regulatory molecules in CLL
}

Justyna A. Wierzbinska ${ }^{1,2,3}$, Reka Toth', Naveed Ishaque ${ }^{3}$, Karsten Rippe ${ }^{3,4}$, Jan-Philipp Mallm³,4 Lara C. Klett ${ }^{2,4}$, Daniel Mertens ${ }^{3,5}$, Thorsten Zenz ${ }^{6}$, Thomas Hielscher ${ }^{7}$, Marc Seifert ${ }^{8}$, Ralf Küppers' ${ }^{8}$, Yassen Assenov ${ }^{1}$, Pavlo Lutsik $^{1}$, Stephan Stilgenbauer ${ }^{9}$, Philipp M. Roessner ${ }^{10}$, Martina Seiffert ${ }^{10}$, John Byrd ${ }^{11}$, Christopher C. Oakes ${ }^{11,12}$, Christoph Plass ${ }^{1,3^{*}}$ and Daniel B. Lipka ${ }^{3,13,14,15^{*}+}$ (I)

\begin{abstract}
Background: In cancer, normal epigenetic patterns are disturbed and contribute to gene expression changes, disease onset, and progression. The cancer epigenome is composed of the epigenetic patterns present in the tumor-initiating cell at the time of transformation, and the tumor-specific epigenetic alterations that are acquired during tumor initiation and progression. The precise dissection of these two components of the tumor epigenome will facilitate a better understanding of the biological mechanisms underlying malignant transformation. Chronic lymphocytic leukemia (CLL) originates from differentiating B cells, which undergo extensive epigenetic programming. This poses the challenge to precisely determine the epigenomic ground state of the cell-of-origin in order to identify CLL-specific epigenetic aberrations.

Methods: We developed a linear regression model, methylome-based cell-of-origin modeling (Methyl-COOM), to map the cell-of-origin for individual CLL patients based on the continuum of epigenomic changes during normal B cell differentiation.

Results: Methyl-COOM accurately maps the cell-of-origin of CLL and identifies CLL-specific aberrant DNA methylation events that are not confounded by physiologic epigenetic B cell programming. Furthermore, Methyl-COOM unmasks abnormal action of transcription factors, altered super-enhancer activities, and aberrant transcript expression in CLL. Among the aberrantly regulated transcripts were many genes that have previously been implicated in $T$ cell biology. Flow cytometry analysis of these markers confirmed their aberrant expression on malignant B cells at the protein level.

Conclusions: Methyl-COOM analysis of CLL identified disease-specific aberrant gene regulation. The aberrantly expressed genes identified in this study might play a role in immune-evasion in CLL and might serve as novel targets for immunotherapy approaches. In summary, we propose a novel framework for in silico modeling of reference DNA methylomes and for the identification of cancer-specific epigenetic changes, a concept that can be broadly applied to other human malignancies.
\end{abstract}

Keywords: DNA methylation, Cell-of-origin, Chronic lymphocytic leukemia, T cell antigens

\footnotetext{
* Correspondence: c.plass@dkfz.de; d.lipka@dkfz.de

${ }^{+}$Christoph Plass and Daniel B. Lipka contributed equally to this work.

${ }^{1}$ Division of Cancer Epigenomics, German Cancer Research Center (DKFZ), Im

Neuenheimer Feld 280, 69120 Heidelberg, Germany

${ }^{3}$ The German Cancer Consortium (DKTK), Heidelberg, Germany

Full list of author information is available at the end of the article
}

(c) The Author(s). 2020 Open Access This article is distributed under the terms of the Creative Commons Attribution 4.0 International License (http://creativecommons.org/licenses/by/4.0/), which permits unrestricted use, distribution, and reproduction in any medium, provided you give appropriate credit to the original author(s) and the source, provide a link to the Creative Commons license, and indicate if changes were made. The Creative Commons Public Domain Dedication waiver (http://creativecommons.org/publicdomain/zero/1.0/) applies to the data made available in this article, unless otherwise stated. 


\section{Background}

In cancer, normal epigenetic patterns are disturbed and contribute to gene expression changes, disease onset, and progression [1]. This seems to be a universal characteristic of all cancers, including chronic lymphocytic leukemia (CLL). CLL originates from rapidly differentiating $B$ cells. Although several mutations creating a preleukemic clone, including variants in SF3B1, NOTCH1, or TP53, have been identified in the hematopoietic stem cell (HSC) compartment of CLL patients, additional genetic or epigenetic driver events are required for full transformation [2]. Normal B cells undergo extensive epigenetic programming during differentiation $[3,4]$. The epigenetic fingerprint of the $B$ cell that has acquired the transforming hit is "frozen" and stably propagated in the leukemic cells [4]. This demonstrates that two factors contribute to the epigenomic landscape of CLL: first, epigenetic patterns that were present in the tumor-initiating $B$ cell at the time of transformation, and second, CLLspecific epigenetic alterations that are acquired during leukemia initiation and progression. For the purpose of this study, we define the cell-of-origin of CLL as the normal B cell differentiation stage with the highest overlap to the CLL methylome. Consequently, the cell-of-origin of CLL represents the differentiation stage at which the clonal B cells deviate significantly from the normal differentiation trajectory and therefore the cell-of-origin defines the first cell that has acquired sufficient oncogenic hits to initiate leukemic transformation [5].

Numerous publications have reported extensive epigenetic alterations in CLL resulting in deregulation of protein coding genes [6-11] or miRNAs [12-19]. In this context, most studies used the epigenome of $\mathrm{CD} 19^{+} \mathrm{B}$ cells as controls, but such an approach neglects the epigenetic programming occurring during $B$ cell differentiation. As a result, the genes found to be deregulated mainly reflected the changes occurring during normal $\mathrm{B}$ cell differentiation rather than CLL-specific pathogenic events. Refined analyses should aim at discriminating between epigenetic changes occurring during normal B cell differentiation and CLL-specific epigenetic aberrations. Here we outline a novel framework for cancer methylome analysis, termed methylome-based cell-of-origin modeling (Methyl-COOM). We show how MethylCOOM can be applied to epigenomic datasets from CLL patients to identify disease-specific epigenetic events and demonstrate its power to detect epigenetically deregulated transcripts which encode for proteins that are involved in immune regulatory processes.

\section{Methods}

Flow cytometry analysis

Patients' samples were obtained from the Department of Internal Medicine III of Ulm University after approval of the study protocol by the local ethics committee according to the Declaration of Helsinki, and after obtaining informed consent of patients. Patients met standard diagnosis criteria for CLL. Patients' characteristics such as age, gender, mutational state, and Binet stage are depicted in Table 1.

Peripheral blood was drawn using ethylenediaminetetraacetic acid (EDTA)-coated tubes (Sarstedt, Nümbrecht, Germany). PBMCs were isolated by Ficoll (Biochrom, Berlin, Germany) density gradient centrifugation. PBMCs were viably frozen and, when needed, thawed and further processed.

After blockade of Fc receptors using Human TruStain $\mathrm{FcX}^{\mathrm{m}}$ (BioLegend, London, UK), $5 \times 10^{6}$ PBMCs were stained with fluorescently labeled antibodies in phosphatebuffered saline (PBS) with addition Fixable Viability Dye eFluor ${ }^{\circledR}$ (Thermo Fisher Scientific, Dreieich, Germany) for $30 \mathrm{~min}$ at $4{ }^{\circ} \mathrm{C}$. Cells were fixed using eBioscience ${ }^{\mathrm{Tm}}$ IC Fixation Buffer (Thermo Fisher Scientific, Dreieich, Germany) for $30 \mathrm{~min}$ at room temperature. The antibodies used are listed in Table 2. If necessary, cells were permeabilized with eBioscience $^{\text {tm }}$ Permeabilization Buffer (Thermo Fisher Scientific) and stained intracellularly for $30 \mathrm{~min}$ at room temperature. CTLA-4 was stained as surface as well as intracellular marker. Samples were stored at $4{ }^{\circ} \mathrm{C}$ in the dark until acquisition. Data was acquired using a BD LSR Fortessa (BD Biosciences, Heidelberg, Germany) FACS analyzer. Flow cytometric data was analyzed using FlowJo X 10.0.7 software (FlowJo, Ashland, OR, USA). Paired Wilcoxon signed-rank test was used to determine statistical significance of changes between CLL B cells and normal B cells.

Table 1 Characteristics of the CLL patients used for flow cytometric analysis

\begin{tabular}{ll}
\hline \# of patients & 7 \\
Age [years] & 57.1 (mean) \\
& 52 (median) \\
Sex & $5 / 7$ male \\
& $2 / 7$ female \\
Prior therapies & $7 / 7$ no prior treatment \\
Binet stage & $7 / 7 \mathrm{~A}$ \\
& $0 / 7 \mathrm{~B}$ \\
& $0 / 7 \mathrm{C}$ \\
IGHV status & $6 / 7$ mutated \\
& $1 / 7$ unmutated \\
Genetics (FISH) & $1 / 7$ Trisomy 12 \\
& $5 / 7$ del(13q) \\
& $1 / 7$ no aberration \\
TP53 mutation status & $4 / 7$ WT \\
& $3 / 7$ not tested \\
\hline
\end{tabular}


Table $\mathbf{2}$ List of FACS antibodies and reagents

\begin{tabular}{|c|c|c|c|}
\hline Reagent & Clone & Supplier & Cat \# \\
\hline APC anti-human CD5 & $\mathrm{UCHT} 2$ & BioLegend & 300612 \\
\hline eBioscience ${ }^{T M}$ CD152 (CTLA-4) PerCP-eFluor 710 & 14D3 & Thermo Fisher Scientific & 46-1529-42 \\
\hline eBioscience $^{T M}$ CD276 (B7-H3) PE-Cyanine7 & $7-517$ & Thermo Fisher Scientific & $25-2769-41$ \\
\hline eBioscience ${ }^{T M}$ Fixable Viability Dye eFluor $r^{T M} 506$ & & Thermo Fisher Scientific & 65-0866-14 \\
\hline eBioscience $^{T M}$ IC Fixation Buffer & & Thermo Fisher Scientific & $00-8222-49$ \\
\hline eBioscience ${ }^{\text {TM }}$ Permeabilization Buffer (10X) & & Thermo Fisher Scientific & 00-8333-56 \\
\hline eBioscience ${ }^{\text {TM }}$ TIGIT PE-Cyanine7 & MBSA43 & Thermo Fisher Scientific & $25-9500-42$ \\
\hline Human TruStain FcX'⿳一由工 (Fc Receptor Blocking Solution) & & BioLegend & 422302 \\
\hline PE anti-human CD85k (ILT3, LILRB4) Antibody & ZM4.1 & BioLegend & 333007 \\
\hline PE/Dazzle ${ }^{\text {TM }} 594$ anti-human CD19 Antibody & HIB19 & BioLegend & 302252 \\
\hline PerCP/Cyanine5.5 anti-human CD2 & RPA-2.10 & BioLegend & 300215 \\
\hline
\end{tabular}

\section{Analysis of RNA-seq/sncRNA-seq data}

Expression data (RNA-Seq) from CLLs were obtained from our previous study [4]. RNA-Seq data from normal $B$ cells was obtained from International Cancer Genome Consortium (ICGC). Reads per kilo base per million mapped reads (RPKM) normalized values were used for the comparison of gene expression levels. sncRNA-seq data from CLLs was obtained from our previous study [20]. Differential miRNA expression was assessed using normalized counts, reads per million (RPM).

\section{Analysis of $450 \mathrm{~K}$ methylome array data}

$450 \mathrm{~K}$ data from $\mathrm{B}$ cells was obtained from Oakes et al. [4]. CLL $450 \mathrm{~K}$ data for the discovery and validation cohorts were both obtained from previous studies $[4,21]$. The analysis of $450 \mathrm{~K}$ data was performed using RnBeads software [22]. Both datasets (normal B cells and CLLs) were processed simultaneously (Additional file 11). Briefly, raw $450 \mathrm{~K}$ data for both CLL and healthy B cell sample sets were normalized by the BMIQ method [23] without the background subtraction. The probes overlapping SNPs and the X and $\mathrm{Y}$ chromosomes were removed, and the remaining probes $(n=464,743 \mathrm{CpGs})$ were considered for the downstream analysis ("Cell-of-origin-based methylome analysis (Methyl-COOM)" section).

We studied the DNA methylation programming during normal B cell differentiation, using six discrete B cell subpopulations including naïve to mature B cells: referred to as naïve $B$ cells (NBCs), germinal center founder cells (GCFs), low- and intermediate-memory B cells (loMBCs, intMBCs), splenic marginal zone B cells (sMGZs), and high maturity memory B cells (hiMBCs). DNA methylomes from 2 to 4 donors per normal B cell subpopulation. In addition, 34 CLL samples were analyzed using Illumina 450K Bead Chip arrays.
Cell-of-origin-based methylome analysis (Methyl-COOM)

For analysis, we determined the DNA methylation dynamics during normal B cell differentiation (differentiation axis). Here we assumed that changes in DNA methylation during the cellular differentiation process are reminiscent of the DNA nucleotide changes over the evolutionary time. CpG sites showing a statistically significant gain or loss of methylation of more than $20 \%$ during B cell differentiation defined our set of so-called B cell-specific CpGs ( $n=74,333$ CpGs; Student's $t$ test). A Manhattan distance matrix was calculated and used to build a methylationbased phylogenetic tree of normal B cell differentiation by applying the minimum evolution method (fastme.bal function, R package "ape"; Desper and Gascuel [24]). Each node in the phylogenetic tree corresponds to a certain differentiation stage reached by the $B$ cell. Using this approach, we observed a non-branched differentiation trajectory of normal B cell differentiation. Therefore, we initially used all B cell-specific CpGs to generate a linear regression model of DNA methylation programming during normal $\mathrm{B}$ cell differentiation. Linear behavior between the differentiation stage of every $\mathrm{B}$ cell subset and the methylation profiles at B cell-specific CpGs were tested at the single $\mathrm{CpG}$ level using F-test. The majority of the $\mathrm{B}$ cell-specific CpGs (79.8\%, $n=59,326 \mathrm{CpGs)}$ showed linear methylation dynamics across the six B cell differentiation states. To exclude a potential bias on differentiation stage assignment, we re-created both the phylogeny and the regression model of normal B cell differentiation, this time using the linearly behaving B cell-specific CpGs, only. The final regression model was designed to infer DNA methylation levels of all CpGs included in our analysis.

Next, we mapped all CLL samples onto the normal B cell differentiation trajectory in order to infer the closest virtual normal B cell methylome (cell-of-origin) defined as the position of the closest normal $\mathrm{B}$ cell node in the phylogenetic tree. Then, we applied the linear regression 
model to infer the DNA methylation levels for each CpG site in the putative cell-of-origin for every patient, according to the formula:

$$
M=\alpha+\beta \times \text { d.s. }
$$

where $M$ denotes the calculated beta methylation value for a CpG site of cell-of-origin, d.s. denotes the differentiation stage (defined as the distance between the NBC and the cell-of-origin nodes as determined by the phylogenetic analysis), $\beta$ denotes the slope of the regression line, and $\alpha$ denotes the vertical ( $y$-axis) intercept.

To test our cell-of-origin assignment, we applied a cross-validation model on our phylogenetic analysis. The patient cohort was repeatedly divided into two subgroups; $70 \%$ and $30 \%$ (5000 repetitions). To minimize the likelihood of selecting the same sample multiple times, a random sampling was allowed in the $70 \%$ group, while sample replacement was restricted only to the $30 \%$ group. Using this approach, we observed that our original cell-of-origin is located between interquartile ranges of the cross-validation assignments, confirming the robustness of the cell-of-origin definition (Additional file 1: Figure S2f).

\section{Identification of CLL-specific DNA methylation}

Subsequently, the inferred DNA methylome of the cellof-origin was used as a reference to determine aberrantly methylated CpG sites in each sample. Disease-specific CpGs were defined as sites with significant deviation from the expected methylation levels as compared to the corresponding cell-of-origin.

\section{Sites with epigenetic $B$ cell programming}

Sites undergoing epigenetic B cell programming (i.e., B cell-specific CpGs) could still show disease-specific methylation events if their actual methylation status massively deviates from what would be expected based on the regression model (sites with "epigenetic B cell programming"). We used a conservative cut-off of more than $20 \%$ methylation loss (class A) or gain (class B) relative to the calculated cell-of-origin methylation value ( $M$ value) in at least $75 \%$ of the CLL patients.

\section{Sites without epigenetic $B$ cell programming}

Sites with no epigenetic B cell programming (i.e., non-B cell-specific CpGs) were defined to have CLL-specific aberrant DNA methylation if they displayed either methylation loss (class C) or gain (class D) of more than $20 \%$ relative to the cell-of-origin in at least $75 \%$ of the CLL patients.

\section{Identification of CLL-specific protein-coding genes}

To identify CLL-specific protein-coding genes, disease-specific methylation events were overlapped with promoter regions $(-2.5 \mathrm{~kb},+0.5 \mathrm{~kb}$ to TSS $)$ of protein-coding genes. Next, correlation between aberrant DNA methylation and gene expression was determined (Pearson correlation test, $p$ value $<0.05$; correlation coefficient $\leq 0.7)$. A full list of identified CLL-specific protein-coding genes is available in Additional file 2: Table S1.

\section{Identification of CLL-specific SE-associated genes}

To identify CLL-specific super-enhancer (SE)-associated genes, SE data from DKFZ PRECiSe consortium was used [25]. All statistically significant, differential super-enhancers being gained in CLLs ("gained", $p<$ $0.05, \mathrm{FC}>0$ ) and consensus super-enhancers shared between normal B cells and CLLs ("stable") were used for the analysis. Firstly, SEs were associated with the closest gene in the vicinity. CLL-specific methylation events were then overlapped with SE coordinates. Next, correlation between aberrant DNA methylation in SE region and gene expression of the SE-closest gene (Pearson correlation test, $p$ value $<0.05$; correlation coefficient $\leq 0.7)$ was used to identify CLLspecific super-enhancer (SE)-associated genes. A full list of identified SE-associated genes is available in Additional file 3: Table S2.

\section{Super-enhancer (SE) enrichment analysis}

For the super-enhancer enrichment analysis two sets of super-enhancers were used, SE data from DKFZ PRECiSe consortium [26] and SE data from Ott et al. [27]. From DKFZ PRECiSe consortium all statistically significant, differential super-enhancers being gained in CLLs ("gained", $p<0.05, \mathrm{FC}>0$ ) and consensus superenhancers shared between normal B cells and CLLs ("stable") were used for the analysis. From Ott et al. paper, a unified SE region was created using reduce function in GenomicRanges package, providing a SE data from individual CLL patients $(n=18)$. All CpG probes present on the $450 \mathrm{~K}$ array were used as a background in the enrichment analysis.

\section{Identification of micro-RNA promoters}

To identify miRNA promoters, the promoter segmentation data from CLLs (DKFZ PRECiSe consortium; promoter segmentation data is deposited under GSE113336; raw ChIP-seq data can be found in the European Genome-phenome Archive under the accession number EGAS00001002518) and normal cell lines (Encyclopedia of DNA Elements - ENCODE; ENCODE Mar 2012 Freeze, UCSC accession numbers: wgEncodeEH000784, wgEncodeEH000785, wgEncodeEH000790, 
wgEncodeEH000789, wgEncodeEH000788, wgEncodeEH000786, wgEncodeEH000787, wgEncodeEH000791, wgEncodeEH000792) were used. To define constant promoter segments, the reduce function from the "GenomicRanges" R package was used to create simplified promoter regions, present in all datasets (CLL and ENCODE segmentation data). Putative promoters of primiRNAs were assigned based on their distance to the primiRNA TSSs. The genomic coordinates of pri-miRNAs/ miRNAs were downloaded from miRBase (version 20; v20). Any promoter located within $100 \mathrm{~kb}$ upstream of a pri-miRNA TSS was considered as a putative pri-miRNA promoter. The distance of $100 \mathrm{~kb}$ was chosen based on similar approaches that have been used in the past by Corcoran et al., Fujita et al., and Fukao et al. [25, 28, 29]. The larger distance of putative promoters to pri-miRNA TSSs is especially important in case of intergenic miRNAs, which are originating from intronic sequences and which are considered to be transcribed together with their host gene.

\section{Identification of CLL-specific micro-RNAs}

To identify CLL-specific microRNAs, disease-specific methylation events were overlapped with potential primiRNA promoters. To identify candidate CLL-specific miRNAs, correlation between aberrant DNA methylation and pri-miRNA expression was determined (Spearman correlation test, $p$ value $<0.05$; abs (correlation coefficient $\rho) \geq 0.35$ ). Since many mature miRNAs are derived from the same pri-miRNAs, correlations were calculated using pri-miRNA expression levels determined by sncRNA-seq. A full list of identified CLLspecific microRNAs is available in Additional file 4: Table S3.

\section{Target genes of CLL-specific microRNAs}

To link CLL-specific microRNAs with their pathogenetic effects, two databases of experimentally validated microRNA-target gene interactions were used, TarBase v8.0 and miRTarBase. A full list of experimentally validated CLL-specific microRNA targets is included in Additional file 5: Table S4. To find whether CLL-specific microRNAs are targeting epigenetic regulators, the comprehensive list of epigenetic regulators was used (Additional file 6: Table S5). The list of epigenetic regulators was further used as a query for the list of CLL-specific microRNA targets defined above. The epigenetic regulators targeted by CLL-specific microRNAs are included in the Additional file 7: Table S6.

\section{Transcription factor enrichment analysis}

Transcription factor motif analysis in disease-specific methylation events was performed using HOMER software v4.5 [30] using only the results for the "known motifs" analysis. All CpGs present on the $450 \mathrm{~K}$ array were used as a background, and adjustment for GC and CpG content was used. Furthermore, enrichment of actual binding events of TFs and other DNA-binding proteins was analyzed using available ChIP-seq data from the tier 1 ENCODE cell line GM12878 (for a complete list of datasets used for this analysis, please refer to Additional file 8: Table S7). The ChIP-seq enrichment analysis was performed using the LOLA tool [31] providing all $\mathrm{CpG}$ probes present on the $450 \mathrm{~K}$ array as the "universe". Unsupervised hierarchical clustering and data visualization were performed using $R$.

\section{Results}

\section{Modeling of normal B cell differentiation}

CLL epigenomes are shaped by two major components. The first component constitutes signatures that stem from the leukemia-initiating B cell. The second component is formed by epigenetic alterations acquired during leukemogenesis and progression of the disease. To discriminate these components, we developed an in silico approach to infer DNA methylation dynamics during normal B cell differentiation and to model the epigenome of the cell-of-origin, utilizing previously published Illumina $450 \mathrm{~K}$ array DNA methylome data from six distinct B cell subpopulations [4] and from 34 CLL samples [21] (Fig. 1a). Our approach to this was based on classical phylogeny analysis (minimum evolution method, Desper and Gascuel [24]), which is typically used to reconstruct evolutionary processes based on inherent characters. Similarly to copy number or mutational studies [32, 33], phylogeny analysis on DNA methylation has been used successfully to reconstruct the developmental processes occurring during cell proliferation and differentiation $[4,34]$. Therefore, to model B cell differentiation, we inferred the hierarchical relationship between normal $B$ cell subsets ranging from naïve to memory B cells based on their DNA methylation patterns. The normal B cell methylomes were used to identify CpG sites that show dynamic DNA methylation during $B$ cell differentiation (B cell-specific CpGs; see also "Methods"). A total of 74,333 B cell-specific CpGs were identified ( $\geq 20 \%$ DNA methylation change between naïve and differentiated memory B cells, Student's $t$ test, $p$ value $<0.05[4,35])$. Pairwise Manhattan distances based on DNA methylation profiles at B cell-specific CpGs for normal B cell subsets were used to build a methylation-based phylogenetic tree revealing a non-branched trajectory of B cell differentiation (Additional file 1: Figure S1a). This suggested that linear regression might be suitable to model DNA methylation dynamics. The initial linear regression model of $\mathrm{B}$ cell differentiation considered 
a

\section{Methyl-COOM}

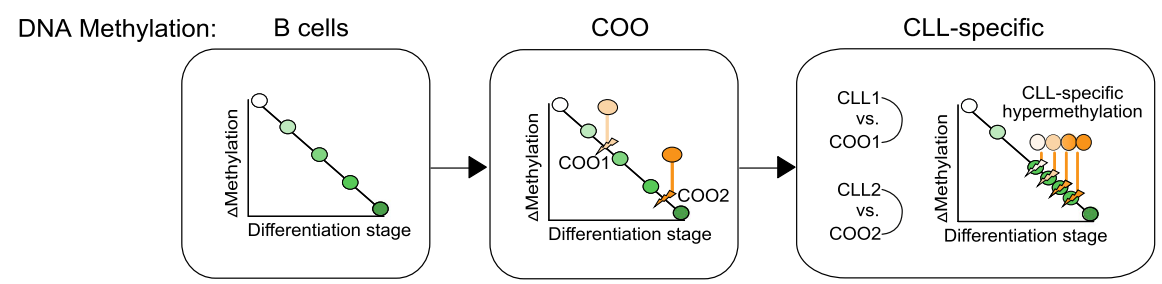

b

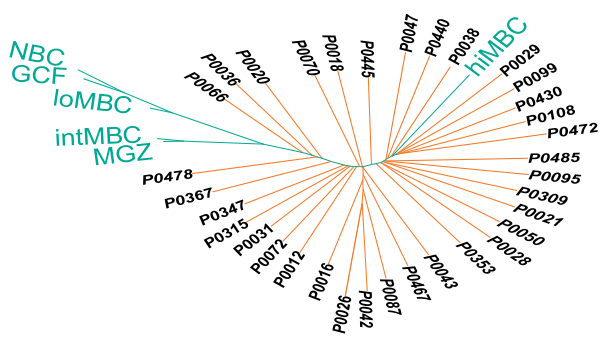

C

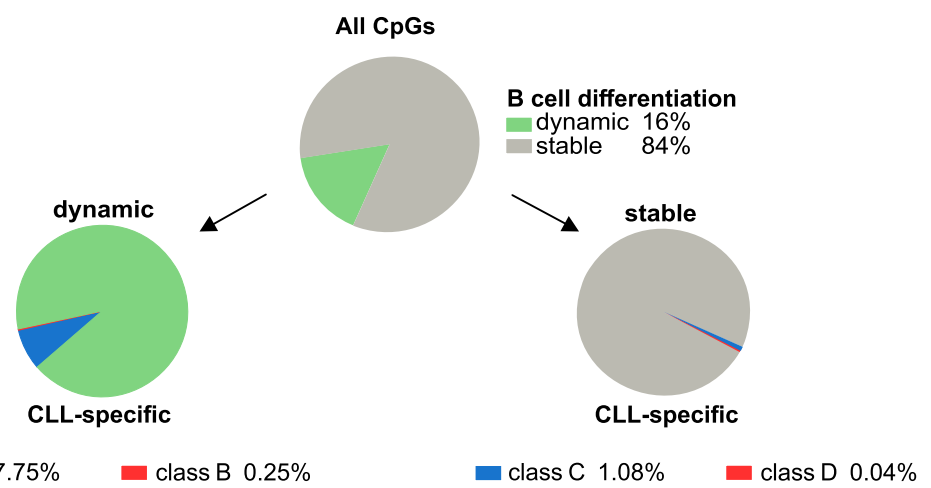

class A $7.75 \%$ class B $0.25 \%$

Sites without epigenetic B cell programming

Sites with epigenetic B cell programming


Fig. 1 (See legend on next page.) 
(See figure on previous page.)

Fig. 1 Identification of CLL-specific DNA methylation events using Methyl-COOM. a Schematic outline of the Methyl-COOM pipeline used for the identification of CLL-specific DNA methylation events. Methylome data of six distinct B cell subpopulations, representing different stages of B cell differentiation were used to infer normal $B$ cell differentiation. A linear regression model was applied to model DNA methylation dynamics during normal B cell differentiation ("DNA methylation: B cells"). DNA methylomes of 34 primary CLL samples were used to identify the closest virtual normal B cell (cell-of-origin; $\mathrm{COO}$ ) based on phylogeny analysis. The linear regression model was then used to infer the DNA methylome of the COO ("DNA methylation: COO"). Next, the DNA methylome of each CLL was compared to the DNA methylome of its COO. CLL-specific aberrant DNA methylation was defined as a significant deviation from the inferred COO methylome ("DNA methylation: CLL-specific"). b Identification of the cell-of-origin in CLL samples using phylogenetic analysis. A phylogenetic tree was generated using a set of linear $\mathrm{CpG}$ sites that show dynamic DNA methylation changes during normal B cell differentiation (linear B cell-specific CpGs, 59,326 CpGs). Pairwise Manhattan distances were calculated between DNA methylation profiles of normal B cells and CLL samples at B cell-specific CpGs and were subsequently used to assign the closest normal (virtual) B cell methylome (location of the node on the phylogenetic tree = differentiation stage of the cell-of-origin) to each CLL case. NBCs - naïve B cells; GCFs - germinal center founder B cells; loMBCs - early non class-switched memory B cells; intMBCs - non class-switched memory B cells; sMGZs - splenic marginal zone B cells; hiMBCs - class-switched memory B cells (mature B cells). CLL samples are depicted in orange color. Normal B cells are represented in green. c Summary of CLL-specific DNA methylation events. Top: pie chart displays the frequency of CpGs that are either dynamic (green) or stable (gray) during normal B cell differentiation. Middle: pie charts depict the frequency of CLL-specific DNA methylation events as fractions of the dynamic (classes A and B; left), and stable (classes C and D; right) sites. Bottom: schematic depicting the classification of CLL-specific DNA methylation events. We identified two groups: "sites with epigenetic B cell programming" and "sites without epigenetic B cell programming." "Sites with epigenetic B cell programming" undergo DNA methylation programming during normal B cell differentiation, encompassing hypomethylation (class A) and hypermethylation events (class B) relative to the DNA methylome of the COO. "Sites without epigenetic B cell programming" are defined as CpG sites without significant DNA methylation changes during normal B cell differentiation and are classified as either hypo- or hypermethylation (classes C and D, respectively). Numbers of CLL-specific DNA methylation events (CLL-specific CPGs) resolved by class are indicated at the bottom

all B cell-specific CpGs. Testing the linearity between the differentiation stage of every normal $B$ cell subset and the methylation profiles at B cell-specific CpGs, revealed that the vast majority of the differentiationspecific CpGs $(79.8 \%, n=59,326$ CpGs) showed linear behavior across all B cell differentiation states (F-test, $p$ value $<0.05$; Additional file 1 : Figure S1b-g, Additional file 9: Table S8). To exclude a potential bias on the model from the non-linear CpG sites, we re-generated both the phylogeny and the regression model of normal $\mathrm{B}$ cell differentiation using only the linearly behaving B cell-specific CpGs.

\section{Identification of disease-specific DNA methylation patterns in CLL}

This B cell differentiation model was applied to a CLL patient cohort $(n=34)$ in order to determine the closest virtual normal B cell methylome (i.e., cell-of-origin or B cell differentiation stage) for each CLL case (Fig. 1b). As expected, our model confirmed that good-prognosis IGHV mutated CLL originates from more mature B cells, as opposed to IGHV unmutated CLL, which develops from more immature $\mathrm{B}$ cells (Additional file 1: Figure S2a-e). Next, we tested the stability of cell-oforigin assignment using a cross-validation model (5000 repetitions; for details see "Methods" section). Using this approach, we observed that the predicted cell-of-origin is located between interquartile ranges of the crossvalidation assignments, confirming the robustness of the cell-of-origin definition (Additional file 1: Figure S2f). The linear regression model was then used to infer DNA methylation levels for all 464,743 CpG sites in the predicted cell-of-origin of every patient. These inferred cell-of-origin methylomes were subsequently used as controls to identify aberrant (i.e., CLL-specific) DNA methylation patterns for each sample individually (see Fig. 1a for a schematic overview of Methyl-COOM). CLL-specific aberrant DNA methylation was defined as CpG sites with $>20 \%$ deviation from the expected DNA methylation level of the cell-of-origin, and which were aberrantly methylated in at least $75 \%$ of patients. This analysis revealed two categories of CLL-specific DNA methylation events; (1) aberrant DNA methylation occurring at sites undergoing epigenetic programming during B cell differentiation ("Sites with epigenetic B cell programming") and (2) aberrant DNA methylation occurring at $\mathrm{CpG}$ sites that normally do not change during B cell differentiation ("Sites with no epigenetic B cell programming") (see Fig. 1c). The first category was further subdivided into class A, showing a loss, and class B, showing a gain of DNA methylation relative to the differentiation stage achieved. The second group of $\mathrm{CpG}$ sites without DNA methylation programming during normal B cell differentiation was subdivided into class $C$ and class $\mathrm{D}$ displaying hypo- and hypermethylation, respectively (Fig. 1c). Overall, only $2.2 \%$ of all CpG sites (10,335 CpGs) represented on the $450 \mathrm{~K}$ array were affected by disease-specific methylation programming, the majority of which were 'sites with epigenetic B cell programming' (class A \& B, 5940 CpG sites; Fig. 1c, Additional file 10: Table S9). The majority of CLL-specific DNA methylation events were characterized by hypomethylation (9995 hypomethylated CLL-specific CpGs; class A: 5757 CpGs, class C: $4238 \mathrm{CpGs}$ ), while only a small proportion of 
CpGs were hypermethylated as compared to their inferred cell-of-origin (340 hypermethylated CLLspecific CpGs; class B: 183 CpGs, class D: 157 CpGs) (Fig. 1c, Additional file 1: Figure S2g, h).

\section{CLL-specific aberrant DNA methylation patterns are independent of the differentiation stage achieved}

CLL-specific DNA methylation changes were quantified for each $\mathrm{CpG}$ site in each sample as compared to the cell-of-origin and inspected by unsupervised hierarchical clustering. For all classes, consistent patterns of either loss or gain in methylation relative to the cell-of-origin were observed, irrespective of the differentiation stage achieved (Fig. 2a, Additional file 1: Figure S2i). Hypomethylation at class A sites resulted from an exaggerated loss of DNA methylation at sites which show loss of methylation during normal B cell differentiation (Fig. 2b, c, Additional file 1: Figure S2i; class A, hypomethylation). Aberrant hypermethylation observed at class $\mathrm{B}$ sites results from exaggeration of hypermethylation normally occurring during $\mathrm{B}$ cell differentiation, and from failed hypomethylation during normal $\mathrm{B}$ cell programming (Fig. 2b, c, Additional file 1: Figure S2i; class B, hypermethylation). Class $\mathrm{C}$ and class $\mathrm{D}$ sites do not undergo any significant DNA methylation programming during normal B cell differentiation, highlighting the potential importance of these sites for CLL pathogenesis (Fig. 2a-c Additional file 1: Figure S2i; class C, class D). Overall, the observed CLL-specific aberrant methylation patterns are largely independent of the differentiation stage achieved by the CLL cell-oforigin.

\section{CLL-specific DNA methylation affects super-enhancers}

To test for functional implications of CLL-specific DNA methylation events, we tested their enrichment in ENCODE ChromHMM genome segments in the GM12878 lymphoblastoid cell line. Aberrantly methylated $\mathrm{CpG}$ sites from classes $\mathrm{A}, \mathrm{B}$, and $\mathrm{C}$ were enriched for enhancer elements (Fig. 3a). A recent systematic assessment of transcription factor dependencies in CLL has implicated super-enhancer (SE)based transcription factor (TF) rewiring in CLL pathogenesis [27, 37]. In line with this, enrichment of CLL-specific CpGs was detected in SE regions identified in a recently published CLL data set from Ott et al. (Additional file 1: Figure S3a) [27]. Using another SE data set from Rippe and colleagues [26, 38] enabled us to distinguish between SEs that are either present in normal B cells ("stable") or that have been acquired de novo in CLL ("gained"). Enrichment of de novo SEs was found in class A and class $C$ sites (Fig. 3b). De novo SEs overlapping with CLL-specific
CpG sites harbor many known genes with relevance in CLL biology (e.g., CD5, CLLU1, IRF2; Additional file 1: Figure S3b, Additional file 3: Table S2).

\section{CLL-specific DNA methylation differences result from aberrant transcription factor programming}

Recent SE perturbation studies implicated rewiring of TF regulatory circuitries in CLL pathogenesis [27]. These findings motivated us to ask whether CLL-specific DNA methylation patterns would be indicative of aberrant TF programming. To address this hypothesis, we used ATAC-seq to test whether CLL-specific DNA methylation patterns were reflected at the level of chromatin accessibility. Indeed, we found that CLL-specific hypo- and hypermethylation events were associated with inverse changes in chromatin accessibility in CLL as compared to normal B cells (Fig. 3c). These concomitant changes in DNA methylation and chromatin accessibility indicated that CLL-specific DNA methylation patterns reflect global epigenomic changes and further demonstrated that disease-specific DNA methylation changes identify functionally relevant cis-regulatory sequences in CLL. In line with this, transcription factor (TF) binding sites enriched in class A (e.g., IKZF1, BATF, NFAT, EGR1/2) and in class $C$ sequences (e.g., NFAT, EGR1/2, E2A) were predominantly associated with B cell biology, e.g., BATF controlling the expression of activationinduced cytidine deaminase (AID) and of $\mathrm{I}_{\mathrm{H}^{-}} \mathrm{C}_{\mathrm{H}}$ germline transcripts or E2A controlling B cell lineage commitment. This suggested involvement of altered TF binding patterns in CLL pathogenesis: class A CpG sites are characterized by stronger than normal TF binding and class $\mathrm{C}$ sites are likely de novo bound by $\mathrm{B}$ cellspecific TFs (Fig. 3d, e). Class B sites were enriched in motifs for EBF, NKX6-1, and PAX5, but overall the motif enrichment as well as the associated changes in chromatin accessibility were only moderate (Fig. 3c-e). Binding of proteins related to genome architecture (CTCF, RAD21, SMC3) was overrepresented in class D sites (Fig. 3d, e). Aberrant DNA methylation patterns at TF binding sites in CLL might be associated with disturbed TF expression levels. TF expression analysis revealed transcriptional deregulation of MAFB, JUN, KLF14, KLF4, IRF2, and EBF1, none of which showed major changes in their promoter DNA methylation status (Additional file 1: Figure S4a, b). Among the deregulated TFs, EBF1 showed the strongest and most consistent transcriptional deregulation with almost complete loss of expression in CLL samples $(\log 2-\mathrm{FC}-$ 7.98 [CLL - hiMBC]; Additional file 1: Figure S4a). The EBF1 downregulation potentially explains the observed CLL-specific hypermethylation at class B sites, as EBF1 has been shown to possess pioneering activity [39]. 




b
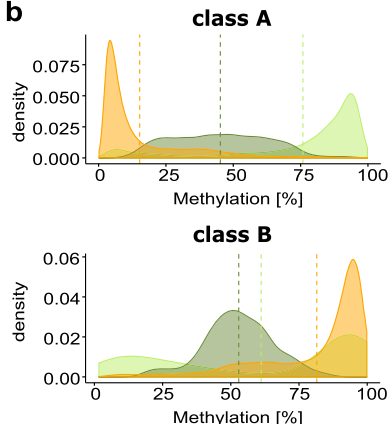

C


class A
hypo
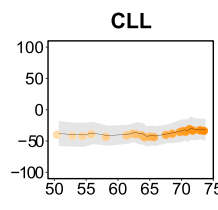

DS [\%]

class B

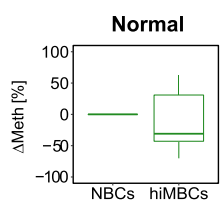



Differentiation Stage (DS) [\%]



Direction (Dir)

hypomethylation

hypermethylation

$\Delta$ Methylation $[\%]$



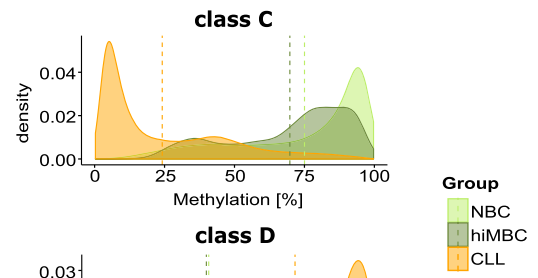

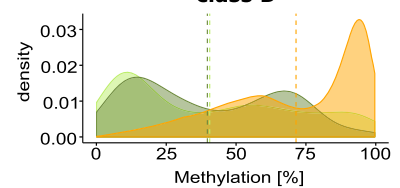

hiMBC
CLL

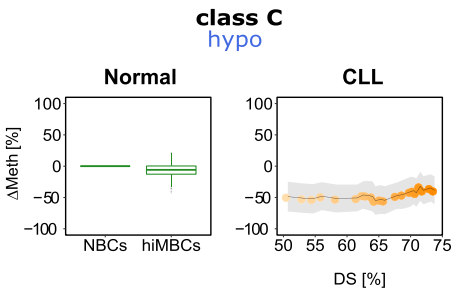

class D
hyper

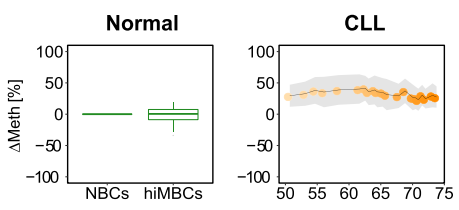

DS [\%]

Fig. 2 Programming of disease-specific DNA methylation patterns in CLL. a Heatmap depicting DNA methylation changes ( $\Delta$ Methylation [\%]) at CLLspecific CpG sites relative to the samples' COO. Unsupervised hierarchical clustering of CLL-specific CpGs, class A and B sites (left), class C and D sites (right). The direction of DNA methylation change (Dir [\%]) is indicated as blue and red bars for hypo- and hypermethylation, respectively, and the numbers of CpG sites plotted are indicated next to the bars. Differentiation stages (DS) are denoted as a color gradient (white-orange), where CLL samples with immature $\mathrm{COO}$ are represented in white and samples with a more mature $\mathrm{COO}$ in orange. DS refers to \% normal differentiation programming achieved (relative to hiMBCs). b Density plots summarizing the distribution of absolute DNA methylation levels for all CLL-specific CpG sites stratified by class (classes A-D). CLL patients (CLL): orange, naïve B cells (NBC): light green, class-switched memory B cells (hiMBC): dark green. c Box plots and ribbon plots displaying the average DNA methylation change for each class of CLL-specific alterations across normal B cells and CLLs. Left (normal): average DNA methylation change ( $\triangle$ Meth) of CLL-specific CpGs during normal B cell differentiation from naïve B cells (NBCs) to class-switched memory B cells (hiMBCs) plotted for all classes (classes A [ $n=5757 \mathrm{CpG}$ sites], B [ $n=183 \mathrm{CpG}$ sites], C $[n=4238 \mathrm{CpG}$ sites], and D $[n=157 \mathrm{CpG}$ sites]). Right (CLL): $\Delta$ Meth for CLLspecific CpGs in CLL. $\triangle$ Meth [\%] is represented as the mean DNA methylation change relative to the expected DNA methylation level of the COO. Standard deviation is depicted as gray shaded ribbons. DS refers to \% normal differentiation programming achieved (relative to hiMBCs) 
a

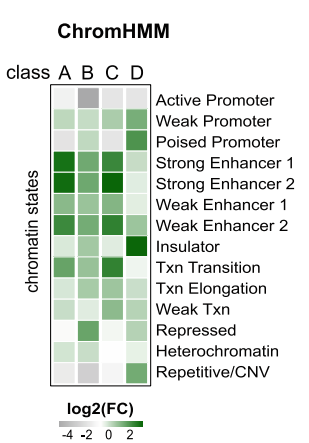

d

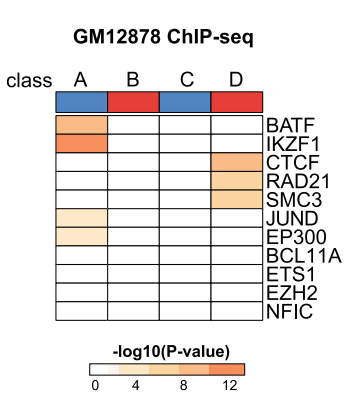

f

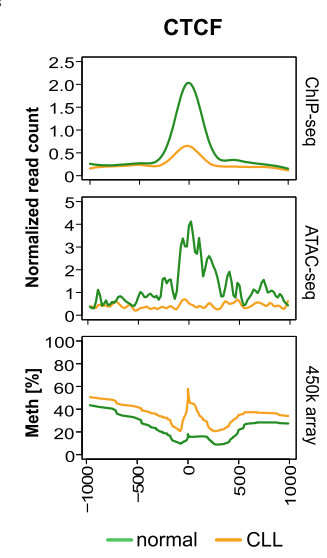

b
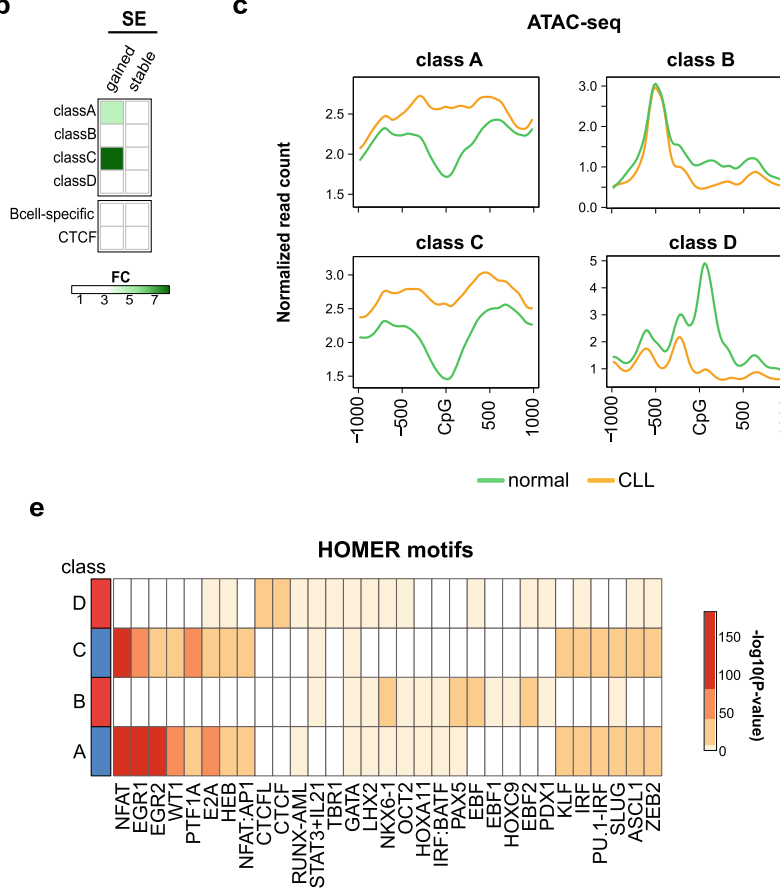

g


Fig. 3 (See legend on next page.) 
(See figure on previous page.)

Fig. 3 CLL-specific DNA methylation differences result from aberrant transcription factor programming. a Enrichment of chromatin states in sequences representing CLL-specific DNA methylation. Chromatin states were defined using the 15-state ChromHMM model from immortalized B cells [36] for CLL-specific methylation sites of the classes A-D. The enrichment in category "Repetitive/CNV" represents the averaged enrichment value of ChromHMM states called "Repetitive/CNV." Log2 fold change (log2 FC) was calculated using all 450K probes as a background. b Enrichment of super-enhancers (SE) in sequences representing CLL-specific DNA methylation. SE were defined as either being gained in CLLS (gained) or consensus between CLLs and B cells (stable). Fold change (FC) was calculated using all 450K probes as a background. c ATAC-seq read density (normalized read counts $\times 10^{-3}$ ) at CLL-specific CpG sites $( \pm 1 \mathrm{~kb})$ for categories of classes $A, B, C$, and D. CLL samples $(n=18)$ are represented in orange, normal CD19+ B cells $(n=3)$ in green. Transcription factor enrichment analysis using ENCODE ChIP-seq peaks from the B-cell lymphoblastoid cell line, GM12878. Displayed are - $\log _{10}$ ( $p$ values) resulting from Fisher's exact test with false discovery rate correction. e Transcription factor motif enrichment analysis using HOMER. The top 10 most enriched TF motifs for each class are displayed. The colors represent $-\log _{10}(p$ values) derived from a cumulative binomial distribution function as implemented in HOMER. $\mathbf{f}$ ATAC-seq \& ChIP-seq read density (normalized read counts $\times 10^{-3}$ ) and DNA methylation profiles at class D CpGs co-locating with CTCF motifs (23 CpGs) ( $\left.\pm 1 \mathrm{~kb}\right)$. CLL samples ( $n=7$ CTCF ChIP-seq, $n=18$ ATAC-seq) are represented in orange, normal CD19+ B cells $(n=4$ CTCF ChIP-seq, $n=3$ ATAC-seq) in green. g Locus plots of exemplary genes associated with CTCF/class D events. Locus plots include data from CTCF ChIP-seq on normal B cells (red) and CLL (blue); ATAC-seq on normal B cells (green) and CLL (purple); RNA-seq on NBC (light green), hiMBC (dark green) and CLL (orange). The class D CpGs are annotated in red

Similarly, upregulation of KLF4, JUN, and IRF2 (Additional file 1: Figure S4a) could explain hypomethylation programming observed at class $\mathrm{A}$ and $\mathrm{C}$ CpG sites as all of these TFs have been reported to possess pioneering activity [40-42].

\section{Class $D$ hypermethylation is associated with reduced CTCF binding and potentially deregulates expression of neighboring genes}

The enrichment of CTCF binding sites and motifs as well as the enrichment of ChromHMM insulator regions (Fig. 3a, d, e) led us to investigate the effects of aberrant CTCF binding in CLL in more detail. We found that class D sites had lower CTCF occupancy and reduced chromatin accessibility in CLL samples as compared to normal B cells (Fig. 3f) while globally, these patterns were identical (Additional file 1: Figure S5a, b). The differences in CTCF binding were associated with changes in gene expression of neighboring genes (Fig. 3g). This further highlights the importance of aberrant CTCF binding at class $\mathrm{D}$ CpGs and might point towards a novel pathogenetic mechanism in CLL. Unfortunately, the low absolute number of class D sites does not allow a comprehensive analysis of associated gene expression changes and further studies involving whole-genome bisulfite sequencing will be required to systematically address this observation.

\section{Identification of epigenetically deregulated transcripts in}

\section{CLL}

The promoter DNA methylation status is widely used as a marker for gene regulation and significant correlation of promoter DNA methylation with gene expression has been demonstrated before [12, 43-45]. Previous studies in CLL identified many epigenetic events potentially deregulating the expression of protein-coding genes and miRNAs. However, all of the work published so far used $\mathrm{CD}_{19^{+}} \mathrm{B}$ cells as controls to call aberrant DNA methylation $[6,9,11$, 46-54]. To stress the importance of using appropriate controls to delineate disease-specific DNA methylation events, we compared our cell-of-origin model to the classical approach using bulk $\mathrm{CD} 19^{+} \mathrm{B}$ cells as a reference. We correlated DNA methylation levels of all aberrant promoter $\mathrm{CpGs}$ with gene expression. The classical approach resulted in a $\sim 1.5$-fold overcalling of epigenetically deregulated protein-coding genes (Additional file 1: Figure S6a). For miRNAs, this difference was even more pronounced (about five- to sevenfold; Additional file 1: Figure S6b). Interestingly, previously identified differentially methylated promoters of TCL1, HOXA4, TWIST2, or $D A P K 1$ did not pass the stringent filtering criteria of our correlation analysis. This suggested that applying Methyl-COOM results in the identification of a more relevant set of epigenetically deregulated candidate genes.

Using the cell-of-origin model, correlation between promoter DNA methylation and miRNA expression levels identified 8 CLL-specific miRNAs (Fig. 4a, b). Seven out of these miRNAs have been demonstrated to regulate epigenetic key players, and, even more importantly, they regulate genes that have been shown to be recurrently mutated in CLL, namely ARID1A, ASXL1, CHD2, SETD1A, SETD2, and KMT2D. Reasoning that miRNA binding to their target genes results in gene expression changes, we compared expression levels between miRNAs and their target genes in CLL and normal B cells. Indeed, concordant with the pattern of miRNA promoter hypomethylation and subsequent upregulation of miRNA transcript levels, we found that known target genes of CLL-specific miRNAs were significantly downregulated in CLL as compared to normal B cells while non-target genes were unaffected (Fig. 4c). 
A similar correlation analysis on protein-coding genes revealed statistically significant correlations between DNA methylation and gene expression for 491 (class A), 20 (class B), 390 (class C), and 20 (class D) genes. The majority of correlations observed were negative (i.e., a decrease in DNA methylation was associated with an increase in gene expression and vice versa; Additional file 1: Figure S6c), and, as expected, the negative correlation with gene expression was most unambiguous for hypermethylation events (59\% class A, $95 \%$ class B, $70 \%$ class C, $85 \%$ class D; Fig. 5a, Additional file 1: Figure S6d). A detailed analysis of the top correlating genes (Pearson correlation test, $p$ value $<0.05$; correlation coefficient $\leq 0.7$ ) encompassing 102 transcripts demonstrated a tight link between CLL-specific aberrant DNA methylation and the expression levels of the corresponding genes (Fig. 5b; Additional file 1: Figure S6a). Normal B cell differentiation-related epigenetic and transcriptional changes were exaggerated in class $\mathrm{A}$ and $\mathrm{B}$ whereas the changes detected in classes $\mathrm{C}$ and $\mathrm{D}$ were observed exclusively in CLL. Aberrantly methylated CpGs of classes A and $\mathrm{C}$ converged in promoters of 12/102 transcripts (TIGIT, SH3D21, LAX1, LILRB4, CD5, NOD2, POLR3GL, IGFBP4, ZAP70, KSR2, XXYLT1-AS2, and LAG3), highlighting the potential functional relevance of the associated genes in CLL pathogenesis. In order to validate our findings, we applied Methyl-COOM to 107 CLL samples that have been published previously by Oakes and colleagues (Additional file 1: Figure S7a; [4]). This analysis identified 11,059 CLL-specific CpGs, of which 8440 (76\%) overlapped with the 10,339 CpGs identified in our discovery cohort (Additional file 1: Figure S7b). Furthermore, CLL-specific CpGs identified in our validation cohort recapitulated 92/102 (90\%) of the top correlating candidate genes found in the discovery cohort (Additional file 1: Figure S7c).

\section{Epigenetically deregulated transcripts are enriched for $\mathrm{T}$ cell-related and immune-modulating genes}

Some of the top correlating genes have already been implicated to play a role in CLL biology, e.g., ZAP70, CD5, LCK, LAG3, or CLLU1 (Additional file 1: Figure S8a, b), while for others their role in CLL pathogenesis is currently unknown. To gain insights into the potential functional role of these epigenetically deregulated genes, we performed enrichment analysis of known biological functions, interactions, or pathways. MSigDB and GO analysis revealed strong enrichment of gene sets related to immune response, immune system processes, hematopoietic stem cells, CLL, and NOTCH signaling (Additional file 1: Figure S8a, b). Ingenuity Pathway Analysis (IPA) and Metascape analysis resulted in enrichment of T-lymphocyte-related processes (Metascape:
"Reguation of T cell activation," "Reguation of T cell receptor signaling pathway," "T cell costimulation," "T cell differentiation," IPA: "Cell Proliferation of $\mathrm{T}$ Lymphocytes," "T cell homeostasis," "Proliferation of lymphocytes" (Additional file 1: Figure S8a). These findings are in line with recent reports demonstrating that $\mathrm{CD} 8^{+} \mathrm{T}$ cells from patients with chronic lymphocytic leukemia exhibit features of $\mathrm{T}$ cell exhaustion, i.e., lower proliferative and cytotoxic capacity and increased expression of inhibitory receptors (e.g., CTLA-4, TIGIT, Lag3, PD-1), suggesting both CLL and T cell-specific changes leading to decreased ability to eliminate malignant cells [55-58].

\section{Epigenetically deregulated transcripts show aberrant protein expression in CLL}

Cancer cells express immune regulatory molecules that might represent potential targets for novel immunotherapies. These proteins modulate the activity of tumorinfiltrating immune cells and mediate immune-escape of tumor cells. Among the epigenetically deregulated genes we identified several with immune regulatory function. Therefore, we aimed to determine whether these are also aberrantly expressed at the protein level in CLL cells. We selected five candidates from the list of top correlated genes which are known to be involved in lymphocyte/T-lymphocyte-related processes (TIGIT, CTLA-4, CD276, LILRB4, and CD2; Fig. 6a). Flow cytometry was utilized for the differential analysis of protein expression in malignant $\left(\mathrm{CD} 19^{+} \mathrm{CD}^{+}\right)$and normal $\left(\mathrm{CD} 19^{+} \mathrm{CD} 5^{-}\right) \mathrm{B}$ cells of 7 CLL patients' blood samples (gating strategy in Additional file 1: Figure S9a). We found that CTLA-4, TIGIT, LILRB4, and CD276 showed statistically significant increased expression in malignant B cells as compared to normal B cells (CTLA-4, $p$ val $=0.047$; TIGIT, $p$ val $=0.016 ;$ CD276, $p$ val $=0.016$; LILRB4, $p$ val $=0.016$ [Wilcoxon paired signed-rank test]), while CD2 surface expression was not detectable neither in normal nor CLL B cells (Fig. 6b; Additional file 1: Figure S9b). Despite the fact that the functional relevance of some of these aberrantly expressed proteins (TIGIT, CD276, or LILRB4) still remains to be elucidated in the context of CLL, our observation is of particular interest for the development of new therapeutic strategies in CLL. Options to interfere with the signaling of these receptors are currently investigated as potential novel therapeutic strategies in several cancer entities.

\section{Discussion}

Applying Methyl-COOM analysis to CLL cells, we identified a number of microRNAs and protein-coding genes that are epigenetically deregulated and validated the CLL-specific epigenetic deregulation for the vast majority of target genes in an independent patient cohort. These epigenetically deregulated transcripts are likely 


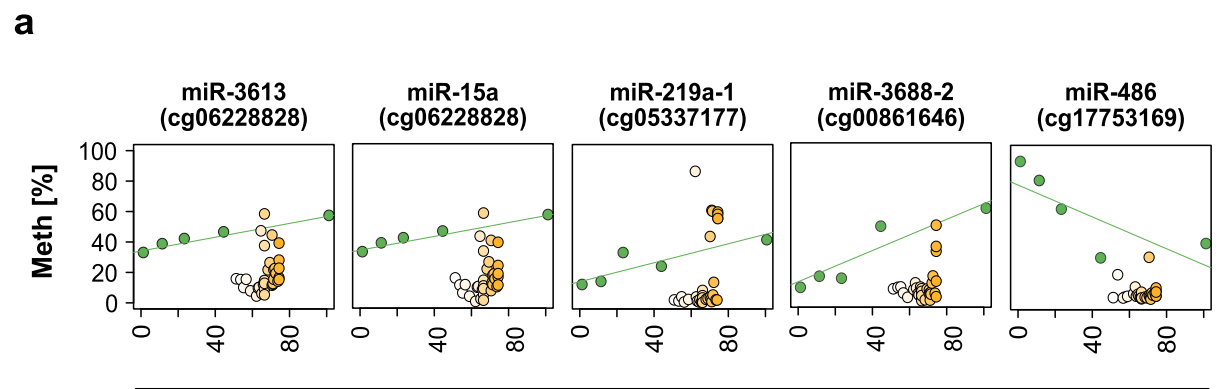

DS [\%]

b

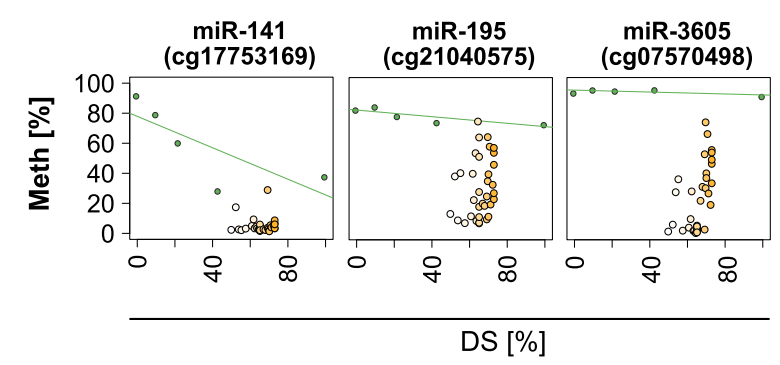

C

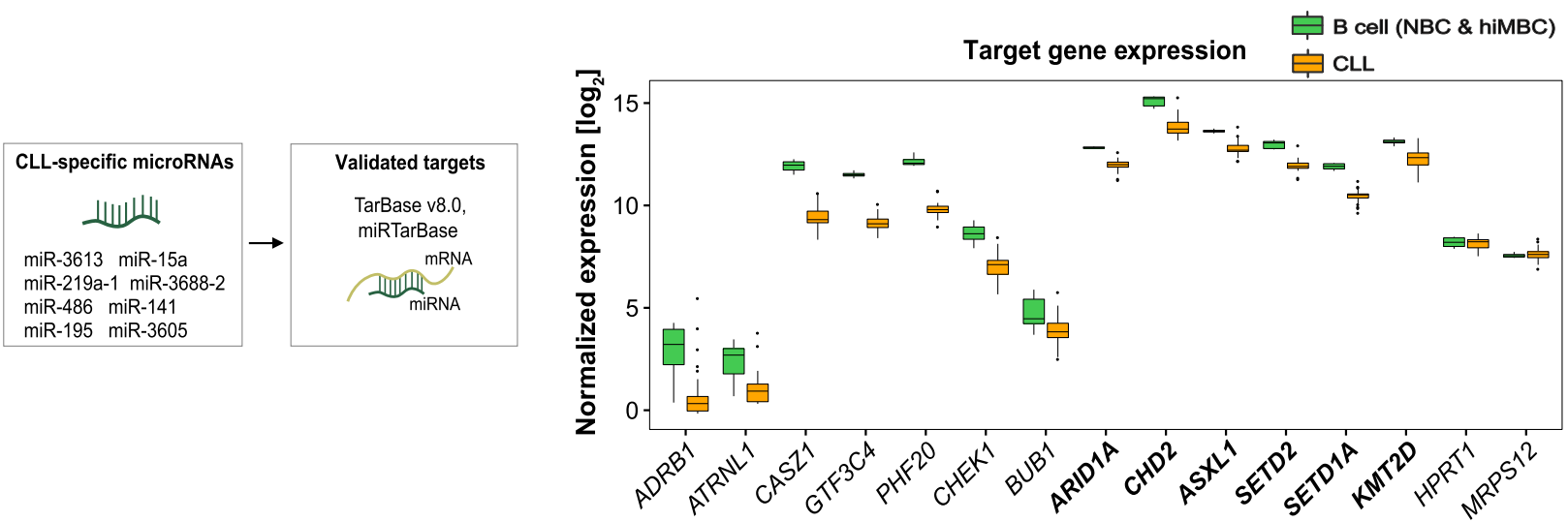

Fig. 4 microRNAs associated with CLL-specific DNA methylation. a Candidate CLL-specific microRNAs deregulated by class A events in their promoter regions. Epigenetic programming during normal B cell differentiation is represented as a green line. Average DNA methylation values are represented as dots; normal B cell subpopulations (green dots); CLL samples (white-orange dots). The $y$-axis represents DNA methylation levels (\%), while the $x$-axis depicts the differentiation stage of normal B cell subpopulations and of CLL samples relative to hiMBCs (DS). b Candidate CLL-specific microRNAs deregulated by class C events in their promoter regions. Epigenetic programming during normal B cell differentiation is represented as a green line. Average DNA methylation values are represented as dots; normal B cell subpopulations (green dots); CLL samples (white-orange dots). The $y$-axis represents DNA methylation levels (\%), while the $x$-axis depicts the differentiation stage of normal $B$ cell subpopulations and of CLL samples relative to hiMBCs (DS). c CLL-specific microRNAs target epigenetic regulators. Left panel: schematic outline of microRNA-target gene prediction. Two databases of experimentally validated targets of microRNAs, TarBase v8.0 and miRTarBase, were used to define a set of CLL-specific microRNA targets. Right panel: normalized gene expression levels (rlog normalized) of epigenetic regulators being targeted by CLL-specific microRNAs as well as gene expression levels of non-target genes (negative controls; HPRT1 and MRPS12) are shown. Recurrently mutated epigenetic regulators in CLL are presented in bold. Statistical significance of expression change between normal $B$ cells (NBCs, hiMBCs) and CLLs was tested using Wilcoxon rank sum test ( $p$ values: ARDB1 $=0.002 ;$ ATRNL1 $=0.0013 ;$ CASZ1 $=0.000014 ;$ GTF3C4 $=$ $0.000014 ;$ PHF20 = 0.000014; CHEK1 = 0.000025; BUB1 = 0.007; $A R I D 1 A=0.000014 ; C H D 2=0.00003 ;$ ASXL1 $=0.00005 ;$ SETD2 $=0.00002 ;$ SETD1A $=$ $0.000014 ; K M T 2 D=0.00007 ;$ HPRT1 $=0.43$, MRPS12 $=0.45)$

involved in the pathogenesis or maintenance of CLL and are functionally enriched for immune system- and lymphocyte-related processes. The expression levels of these transcripts are very low in normal B cells, which is in stark contrast to the strong overexpression observed in CLL cells. These epigenetically deregulated transcripts are further expressed and detectable on the surface of malignant $\mathrm{B}$ cells. CLL patients are known to 

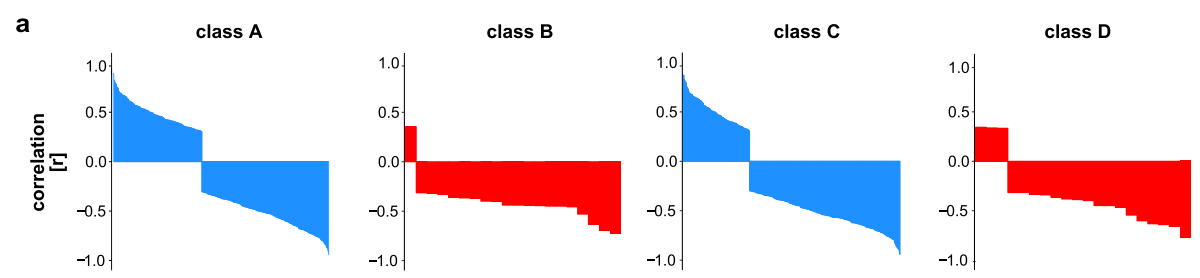

b
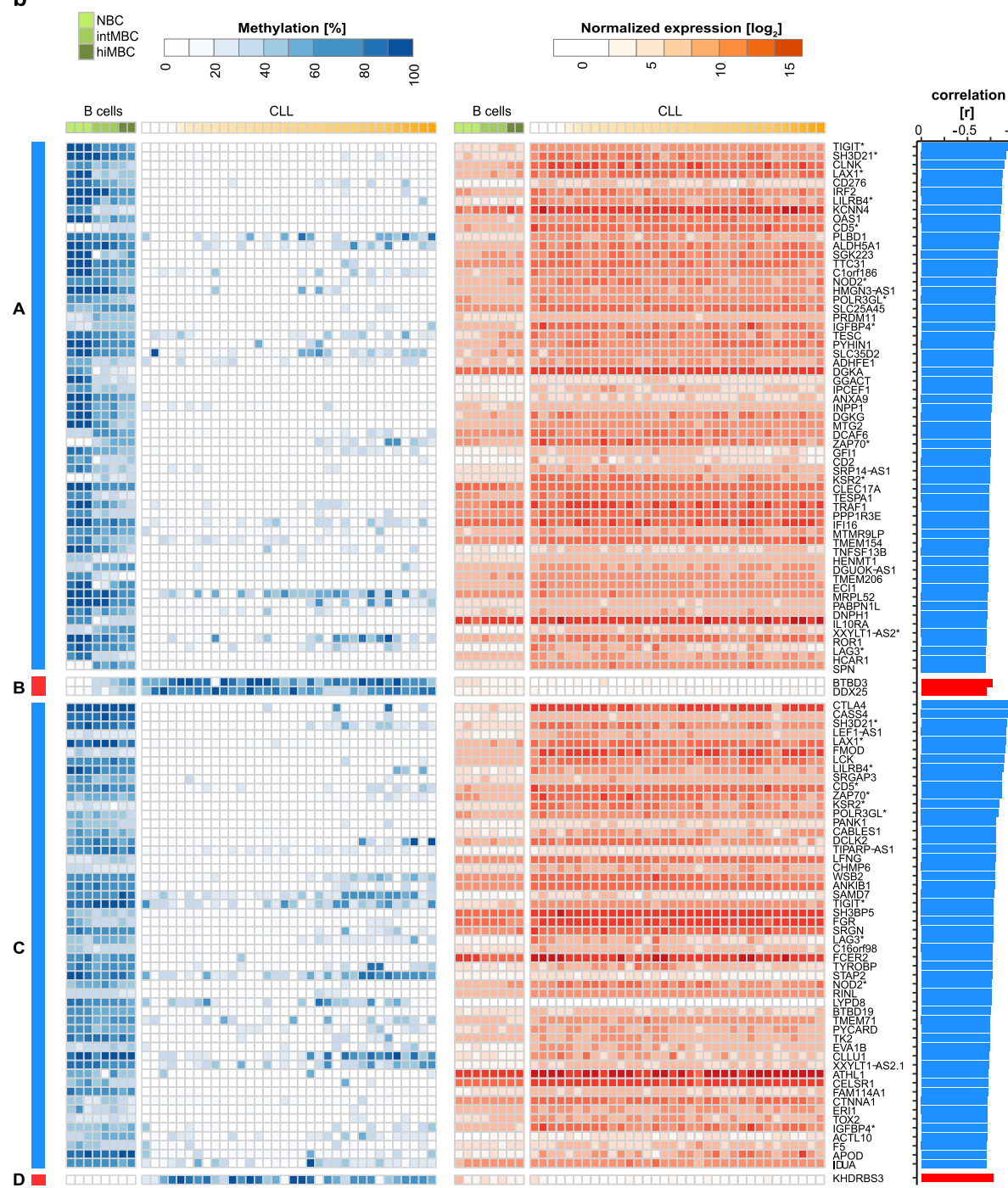

Fig. 5 Protein-coding genes associated with CLL-specific aberrant DNA methylation. a Waterfall plots summarizing the correlation coefficients [r] between DNA methylation in the promoters and gene expression profiles of protein-coding genes for each class of CLL-specific alterations (classes A-D). The direction of DNA methylation change is indicated in blue and red for hypo- and hypermethylation, respectively. $\mathbf{b}$ CLL-specific epigenetically deregulated transcripts. Left panel: heatmap depicting absolute DNA methylation levels [\%] at CLL-specific CpG sites (classes A-D) in the promoter regions of protein-coding genes. Samples were sorted according to the differentiation stage. Differentiation stages are denoted as color gradients, CLLs (white to orange), normal B cells (light to dark green). Middle panel: heatmap depicting normalized gene expression levels (rlog normalization) of protein-coding genes in B cells (light to dark green) and CLLs (white to orange). Transcripts enriched for more than one class of CLL-specific events in their promoter regions are marked with asterisks. Right panel: barplots summarizing correlation coefficients [r] from Pearson correlation analysis between DNA methylation at CLL-specific CpGs in the promoter region and protein-coding gene expression levels. The direction of DNA methylation change is indicated in blue and red for hypo- and hypermethylation, respectively 

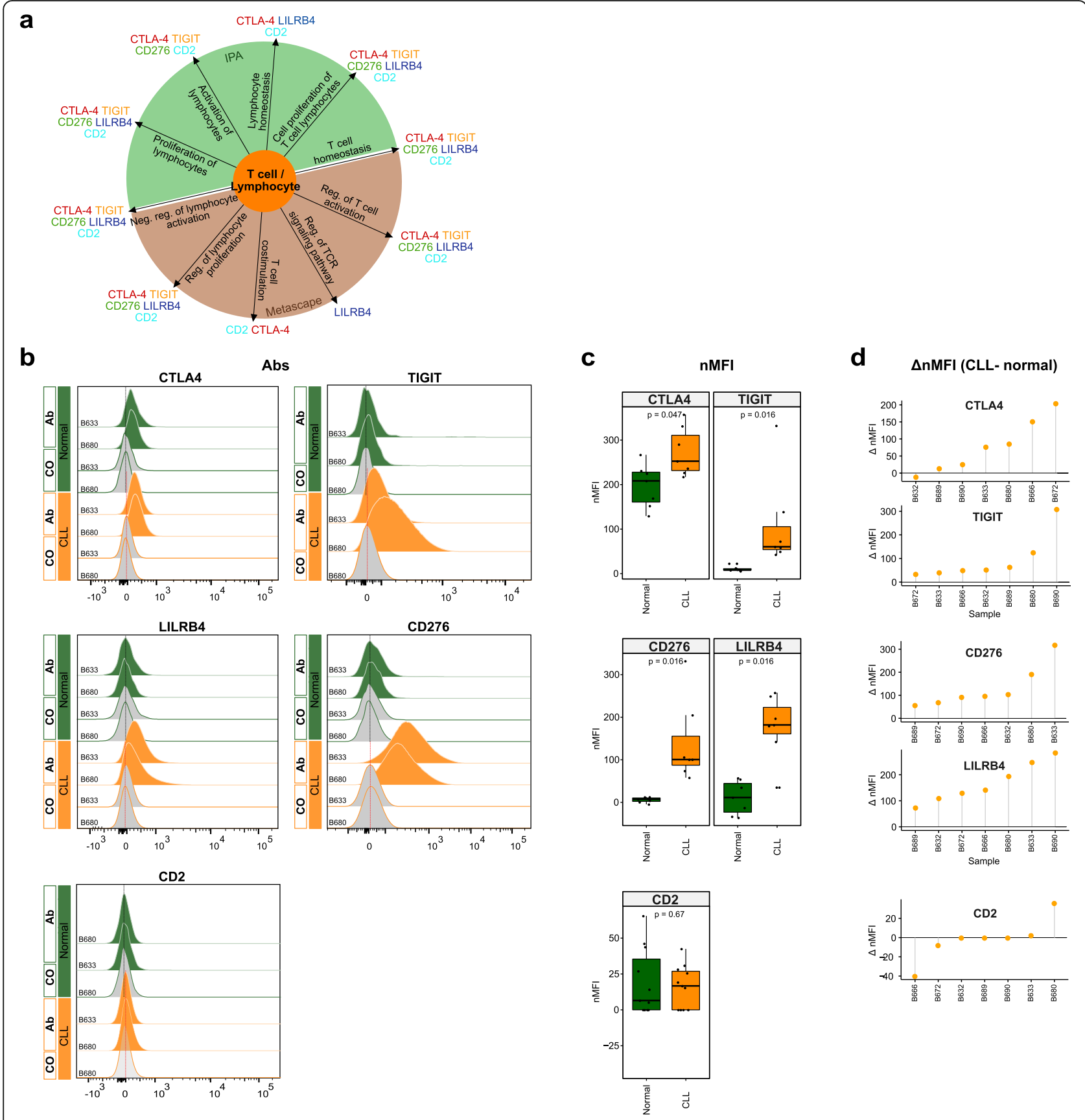

Fig. 6 Flow cytometry analysis of T cell-/lymphocyte-specific markers on normal and malignant B cells from CLL patients. a Summary scheme representing functional implications of CLL-specific candidate genes selected for flow cytometric analysis. b Flow cytometric analysis of expression of CTLA-4, TIGIT, CD276, LILRB4, and CD2 on peripheral blood B cells of CLL patients. The expression was determined for non-malignant B cells ("Normal"; CD19+ CD5 ${ }^{-}$B cells, represented in green) and neoplastic B cells ("CLL", CD19 ${ }^{+} \mathrm{CD}^{+}$B cells, represented in orange) detected in the same samples. "Co," no antibody staining control; "Ab," staining with the antibody of interest as indicated. c Normalized median fluorescence intensities (target MFI - MFI of negative control [Co]; nMFI). $\mathbf{d} \Delta$ normalized median fluorescence intensities between CLL cells and normal B cells ( $\Delta \mathrm{nMFI}(\mathrm{CLL}-$ normal)) for each patient tested

progressively develop an immunosuppressive state including dysfunctional $\mathrm{T}$ cells [58] and our data suggest that CLL cells contribute to the immunosuppressive microenvironment as well as $\mathrm{T}$ cell exhaustion by expressing immune regulatory molecules. Immune dysregulation is known to worsen over the course of the disease, e.g., effector $\mathrm{T}$ cells are increased in early-stage disease and show progressive accumulation and exhaustion in the late-stage $[58,59]$. This, together with the fact that CLL frequently affects older patients with comorbidities, makes CLL an ideal candidate for the development of effective immunotherapies. CD276, TIGIT, 
and LILRB4 would be of particular interest, since to our knowledge they were not yet considered as immunotherapeutic targets in CLL. TIGIT is a recently identified inhibitory receptor expressed on $\mathrm{T}$ cells and natural killer (NK) cells. In T cells, TIGIT expression inhibits cell proliferation, cytokine production, and $\mathrm{T}$ cell receptor signaling [60]. In tumors, TIGIT is involved in mediating a $\mathrm{T}$ cell exhaustion phenotype, which is manifested by poor effector function of $\mathrm{T}$ cells and, consequently, decreased ability to eliminate tumor cells. In non-Hodgkin B cell lymphomas, PD1- and TIGIT-expressing intratumoral $\mathrm{T}$ cells were shown to mark dysfunctional or exhausted effector T cells [61]. CLL patients with an advanced disease stage display elevated numbers of TIGIT $^{+}$ $\mathrm{CD}^{+} \mathrm{T}$ cells compared to low-risk patients [62]. In preclinical models of colorectal and breast carcinoma, TIGIT blockade was shown to reverse the exhaustion phenotype of cytotoxic $\mathrm{T}$ cells and to inhibit tumor growth [63]. Another immune inhibitory receptor, LILRB4, was reported as tumor-associated antigen that is highly expressed on monocytic AML cells $[64,65]$. It was also reported as a selective marker of neoplastic B cells and HSCs from CLL patients [66]. LILRB4 targeting, either by antibodies or by CAR-T cells, impeded AML development [56, 57]. CD276 overexpression, on the other hand, was linked to anti-apoptosis in colorectal cancer through activation of Jak2-STAT3 signaling pathway, and as a result, increased expression of antiapoptotic protein $\mathrm{Bcl}-2$ [67]. High CD276 expression levels were already linked to poor prognosis in CLL and prostate and pancreatic cancer [68-71]. Altogether, TIGIT, LILRB4, and CD276 represent attractive therapeutic targets for treatment of CLL.

The present study demonstrates that Methyl-COOM delineates cancer-specific DNA methylation patterns and identifies deregulated pathways involved in the pathogenesis or maintenance of CLL. Our work serves as a proof of concept that tracing the cell-of-origin by comparison to normal differentiation trajectories is of great conceptual importance in cancer epigenetics. Identifying the cell-of-origin is not only crucial for the precise analysis of epigenetic data, but it is also important for clinical translation. The cell-of-origin impacts on tumor biology, affects chemo- and radiosensitivity, and influences disease outcome. For instance, studies in a murine model of MLL-rearranged AML have shown that the cell-of-origin can influence the phenotype and the aggressiveness of the resulting leukemia [72]. Likewise, glioma subtypes vary in their response to therapy and share molecular signatures with different normal neural lineages, suggesting a difference in their cellular origin [73-77]. So far, the identification of a cancer's cellular origin is based on genetic lineage-tracing experiments in mice, like the ones from Blanpain and colleagues demonstrating the presence of distinct cells-of-origin for two types of skin cancer [78]. In colorectal cancer, the cell-of-origin has been studied intensively, pointing towards three potential cell types as founder cells: intestinal stem cells [79-83], transit amplifying cells [79, 84], and differentiated villus cells [84]. In most instances, however, the precise cell-of-origin, in which transformation occurs, remains undefined.

Methyl-COOM can, in principle, be applicable to any type of DNA methylation data as a source of epigenetic information. In contrast to previous reports in CLL and other malignancies, epigenetic pathomechanisms were investigated using an approach that systematically avoids confounding factors introduced by epigenome dynamics occurring in the context of physiological differentiation processes. It has been demonstrated that similar concepts apply to other lymphatic neoplasms, e.g., T-ALL, DLBCL, or MCL [85-88]. However, for other tumors, including myeloid malignancies, the knowledge on the cell-of-origin is still scarce. Therefore, beyond the field of CLL research, this study could serve as a template for the analysis of epigenomic data in other cancer entities.

\section{Conclusions}

Our work describes a new analytical framework, MethylCOOM, to delineate cancer-specific DNA methylation patterns, a concept that should, in principle, be applicable to all tumor entities. Using Methyl-COOM, we interrogated DNA methylomes of CLL samples in the context of normal B cell differentiation. This enabled us to unmask abnormal transcription factor and superenhancer activities, as well as to identify aberrant transcript expression in CLL. Furthermore, we were able to demonstrate that epigenetically deregulated transcripts are enriched in immune regulatory molecules which are also expressed at the protein level in CLL cells, suggesting that CLL cells contribute to immunosuppression and $\mathrm{T}$ cell exhaustion by upregulation of immune regulatory molecules. This finding might serve as a starting point for the development of novel therapeutic strategies to overcome immune evasion of CLL cells.

\section{Supplementary information}

Supplementary information accompanies this paper at https://doi.org/10. 1186/s13073-020-00724-7.

Additional file 1: Figures $\mathbf{S 1} \mathbf{-} \mathbf{S 9}$, including supplementary figure legends. Additional file 2: Table S1, list of CLL-specific protein-coding genes. Additional file 3: Table S2, list of super-enhancer-associated protein coding genes.

Additional file 4: Table S3, list of CLL-specific miRNAs.

Additional file 5: Table S4, list of candidate target genes of CLLspecific microRNAs.

Additional file 6: Table S5, list of epigenetic regulators. 
Additional file 7: Table S6, list of epigenetic regulators being targeted by CLL-specific microRNAs.

Additional file 8: Table S7, list of ENCODE TF ChIP-seq datasets. Additional file 9: Table S8, list of linear B cell-specific CpGs. Additional file 10: Table S9, list of CLL-specific CpGs (class A, class B, class C, class D).

Additional file 11: List of datasets used in the article.

\section{Acknowledgements}

We would like to thank Thomas Höfer, Stefan Fröhling, Annika Baude, and Simin Öz for helpful discussions.

\section{Authors' contributions}

J.A.W, C.P., and D.B.L. developed the research concept, designed the analysis workflow and experiments, and collected and interpreted the data. J.A.W., R.T., N.I., T.H., Y.A., and P.L. analyzed data. J.A.W. performed experiments. K.R., J.M., L.K., D.M., T.Z., Marc.S., R.K., S.S., J.B., and C.C.O. provided clinical samples or data. P.M.R. and Mart.S. performed flow cytometry experiments and analyzed data. J.A.W, C.P. and D.B.L. prepared the figures and wrote the manuscript. C.P. and D.B.L. jointly supervised the project. All authors contributed to the writing of the manuscript and approved the final version.

\section{Funding}

This work was supported in part by the PRECISE consortium with funds from the German Federal Ministry of Education and Research (031L0076A), and the Helmholtz Foundation (CP, KR, DM, MartS). Further support came from the German Cancer AID (DKH 70113869 to PL, CP). JW was supported by the Helmholtz International Graduate School for Cancer Research in Heidelberg. The funding bodies had no role in the design of the study, nor in the collection, analysis, and interpretation of data, nor in writing the manuscript.

\section{Availability of data and materials}

The datasets used and analyzed in the current study were published previously as indicated in Additional file 11. The Methyl-COOM framework is accessible via GitHub (https://github.com/justannwska/Methyl-COOM) [36]. Bioconductor http://bioconductor.org/ [89].

LOLA https://bioconductor.org/packages/release/bioc/html/LOLA.html [31]. ENCODE https://www.encodeproject.org/ [90].

HOMER http://homer.ucsd.edu/homer/ [30].

miRTarBase: http://mirtarbase.mbc.nctu.edu.tw/php/index.php [91].

TarBase v8.0 http://carolina.imis.athena-innovation.gr/diana_tools/web/index. php?r=tarbasev8\%2Findex [92].

microRNA.org http://www.microrna.org/microrna/home.do [93].

miRBase v.18.0 http://www.mirbase.org [94]

\section{Ethics approval and consent to participate}

The study was conducted in accordance with the Declaration of Helsinki and was approved by the Ethics Committee Heidelberg (University of Heidelberg, Germany; S-206/2011; S-356/2013) and by the Ethics Committee UIm (UIm University; 130/2002). Samples were taken after patients gave their written informed consent.

\section{Consent for publication}

Not applicable.

\section{Competing interests}

The authors declare that they have no competing interests.

\section{Author details}

${ }^{1}$ Division of Cancer Epigenomics, German Cancer Research Center (DKFZ), Im Neuenheimer Feld 280, 69120 Heidelberg, Germany. ${ }^{2}$ Faculty of Biosciences, Heidelberg University, Heidelberg, Germany. ${ }^{3}$ The German Cancer Consortium (DKTK), Heidelberg, Germany. ${ }^{4}$ Division of Chromatin Networks, DKFZ, Heidelberg, Germany. ${ }^{5}$ Mechanisms of Leukemogenesis, DKFZ, Heidelberg, Germany. ${ }^{6}$ Experimental Hematology Lab, University Hospital Zurich, Zurich, Switzerland. ${ }^{7}$ Biostatistics, DKFZ, Heidelberg, Germany. ${ }^{8}$ Group Molecular Genetics, Essen University Hospital, Essen, Germany. ${ }^{9}$ Department of Internal Medicine, Ulm University, Ulm, Germany. ${ }^{10}$ Division of Molecular Genetics, DKFZ, Heidelberg, Germany. ${ }^{11}$ Department of Internal Medicine,
Division of Hematology, The Ohio State University, Columbus, USA. ${ }^{12}$ Department of Biomedical Informatics, The Ohio State University, Columbus, USA. ${ }^{13}$ Section Translational Cancer Epigenomics, Division of Translational Medical Oncology, German Cancer Research Center (DKFZ), Im Neuenheimer Feld 581, 69120 Heidelberg, Germany. ${ }^{14}$ National Center for Tumor Diseases (NCT), Heidelberg, Germany. ${ }^{15}$ Faculty of Medicine, Medical Center, Otto-von-Guericke-University, 39120 Magdeburg, Germany.

Received: 31 July 2019 Accepted: 11 February 2020

Published online: 18 March 2020

\section{References}

1. Baylin SB, Jones PA. A decade of exploring the cancer epigenome biological and translational implications. Nat Rev Cancer. 2011;11:726-34.

2. Damm F, Mylonas E, Cosson A, Yoshida K, Della Valle V, Mouly E, et al. Acquired initiating mutations in early hematopoietic cells of CLL patients. Cancer Discov. 2014;4:1088-101.

3. Kulis M, Merkel A, Heath S, Queiros AC, Schuyler RP, Castellano G, et al. Whole-genome fingerprint of the DNA methylome during human $B$ cell differentiation. Nat Genet. 2015:47:746-56.

4. Oakes CC, Seifert M, Assenov Y, Gu L, Przekopowitz M, Ruppert AS, et al. DNA methylation dynamics during $B$ cell maturation underlie a continuum of disease phenotypes in chronic lymphocytic leukemia. Nat Genet. 2016;48: 253-64.

5. Visvader JE. Cells of origin in cancer. Nature. 2011;469:314-22.

6. Raval A, Tanner SM, Byrd JC, Angerman EB, Perko JD, Chen SS, et al. Downregulation of death-associated protein kinase 1 (DAPK1) in chronic lymphocytic leukemia. Cell. 2007;129:879-90.

7. Raval A, Byrd JC, Plass C. Epigenetics in chronic lymphocytic leukemia. Semin Oncol. 2006;33:157-66.

8. Claus R, Lucas DM, Ruppert AS, Williams KE, Weng D, Patterson K, et al. Validation of ZAP-70 methylation and its relative significance in predicting outcome in chronic lymphocytic leukemia. Blood. 2014;124:42-8.

9. Claus R, Lucas DM, Stilgenbauer S, Ruppert AS, Yu L, Zucknick M, et al. Quantitative DNA methylation analysis identifies a single CpG dinucleotide important for ZAP-70 expression and predictive of prognosis in chronic lymphocytic leukemia. J Clin Oncol. 2012;30:2483-91.

10. Rush LJ, Raval A, Funchain P, Johnson AJ, Smith L, Lucas DM, et al. Epigenetic profiling in chronic lymphocytic leukemia reveals novel methylation targets. Cancer Res. 2004;64:2424-33.

11. Corcoran M, Parker A, Orchard J, Davis Z, Wirtz M, Schmitz OJ, et al. ZAP-70 methylation status is associated with ZAP-70 expression status in chronic lymphocytic leukemia. Haematologica. 2005;90:1078-88.

12. Baer C, Claus R, Frenzel LP, Zucknick M, Park YJ, Gu L, et al. Extensive promoter DNA hypermethylation and hypomethylation is associated with aberrant microRNA expression in chronic lymphocytic leukemia. Cancer Res. 2012;72:3775-85.

13. Pallasch CP, Patz M, Park YJ, Hagist S, Eggle D, Claus R, et al. miRNA deregulation by epigenetic silencing disrupts suppression of the oncogene PLAG1 in chronic lymphocytic leukemia. Blood. 2009;114:3255-64.

14. Wang LQ, Kwong YL, Kho CS, Wong KF, Wong KY, Ferracin M, et al. Epigenetic inactivation of miR-9 family microRNAs in chronic lymphocytic leukemia--implications on constitutive activation of NFkappaB pathway. Mol Cancer. 2013:12:173.

15. Wong KY, Yim RL, Kwong YL, Leung CY, Hui PK, Cheung F, et al. Epigenetic inactivation of the MIR129-2 in hematological malignancies. J Hematol Oncol. 2013;6:16.

16. Wang LQ, Kwong YL, Wong KF, Kho CS, Jin DY, Tse E, et al. Epigenetic inactivation of mir-34b/c in addition to mir-34a and DAPK1 in chronic lymphocytic leukemia. J Transl Med. 2014;12:52.

17. Deneberg S, Kanduri M, Ali D, Bengtzen S, Karimi M, Qu Y, et al. microRNA$34 \mathrm{~b} / \mathrm{c}$ on chromosome $11 \mathrm{q} 23$ is aberrantly methylated in chronic lymphocytic leukemia. Epigenetics. 2014;9:910-7.

18. Baer C, Oakes CC, Ruppert AS, Claus R, Kim-Wanner SZ, Mertens D, et al. Epigenetic silencing of miR-708 enhances NF-kappaB signaling in chronic lymphocytic leukemia. Int J Cancer. 2015;137:1352-61.

19. Wang LQ, Wong KY, Rosen A, Chim CS. Epigenetic silencing of tumor suppressor miR-3151 contributes to Chinese chronic lymphocytic leukemia by constitutive activation of MADD/ERK and PIK3R2/AKT signaling pathways. Oncotarget. 2015;6:44422-36. 
20. Blume CJ, Hotz-Wagenblatt A, Hullein J, Sellner L, Jethwa A, Stolz T, et al. p53-dependent non-coding RNA networks in chronic lymphocytic leukemia. Leukemia. 2015;29:2015-23.

21. Dietrich S, Oles M, Lu J, Sellner L, Anders S, Velten B, et al. Drugperturbation-based stratification of blood cancer. J Clin Invest. 2018;128: 427-45.

22. Assenov Y, Muller F, Lutsik P, Walter J, Lengauer T, Bock C. Comprehensive analysis of DNA methylation data with RnBeads. Nat Methods. 2014;11:1138-40.

23. Teschendorff AE, Marabita F, Lechner M, Bartlett T, Tegner J, Gomez-Cabrero $D$, et al. A beta-mixture quantile normalization method for correcting probe design bias in Illumina Infinium 450 k DNA methylation data. Bioinformatics. 2013;29:189-96.

24. Desper R, Gascuel O. Fast and accurate phylogeny reconstruction algorithms based on the minimum-evolution principle. J Comput Biol. 2002:9:687-705.

25. Corcoran DL, Pandit KV, Gordon B, Bhattacharjee A, Kaminski N, Benos PV. Features of mammalian microRNA promoters emerge from polymerase II chromatin immunoprecipitation data. PLoS One. 2009;4:e5279.

26. DKFZPRECiSE consortium. DKFZ PRECiSE consortium data resources 2018.

27. Ott CJ, Federation AJ, Schwartz LS, Kasar S, Klitgaard JL, Lenci R, et al. Enhancer Architecture and Essential Core Regulatory Circuitry of Chronic Lymphocytic Leukemia. Cancer Cell. 2018;34:982-995.e7.

28. Fujita $\mathrm{S}, \mathrm{lba} \mathrm{H}$. Putative promoter regions of miRNA genes involved in evolutionarily conserved regulatory systems among vertebrates. Bioinformatics. 2008;24:303-8.

29. Fukao T, Fukuda Y, Kiga K, Sharif J, Hino K, Enomoto Y, et al. An evolutionarily conserved mechanism for microRNA-223 expression revealed by microRNA gene profiling. Cell. 2007;129:617-31.

30. Heinz S, Benner C, Spann N, Bertolino E, Lin YC, Laslo P, et al. Simple combinations of lineage-determining transcription factors prime cisregulatory elements required for macrophage and B cell identities. Mol Cell. 2010;38:576-89.

31. Sheffield NC, Bock C. LOLA: enrichment analysis for genomic region sets and regulatory elements in $\mathrm{R}$ and bioconductor. Bioinformatics. 2016;32: 587-9.

32. Navin N, Kendall J, Troge J, Andrews P, Rodgers L, Mclndoo J, et al. Tumour evolution inferred by single-cell sequencing. Nature. 2011:472:90.

33. $X u X$, Hou $Y$, Yin $X$, Bao L, Tang A, Song L, et al. Single-cell exome sequencing reveals single-nucleotide mutation characteristics of a kidney tumor. Cell. 2012;148:886-95.

34. Brocks D, Assenov Y, Minner S, Bogatyrova O, Simon R, Koop C, et al. Intratumor DNA methylation heterogeneity reflects clonal evolution in aggressive prostate cancer. Cell Rep. 2014;8:798-806.

35. Lipka DB, Witte T, Toth R, Yang J, Wiesenfarth M, Nollke P, et al. RAS pathway mutation patterns define epigenetic subclasses in juvenile myelomonocytic leukemia. Nat Commun. 2017;8:2126.

36. Wierzbisnka JA. Methyl-COOM Framework. Available from: https://github. com/justannwska/Methyl-COOM. Accessed 02 Feb 2020.

37. Lipka DB, Lutsik P, Plass C. From basic knowledge to effective therapies. Cancer Cell. 2018:34:871-3.

38. Mallm J-P, Iskar M, Ishaque N, Klett LC, Kugler SJ, Muino JM, et al. Linking aberrant chromatin features in chronic lymphocytic leukemia to transcription factor networks. Mol Syst Biol. 2019;15:e8339.

39. Boller S, Ramamoorthy S, Akbas D, Nechanitzky R, Burger L, Murr R, et al. Pioneering activity of the C-terminal domain of EBF1 shapes the chromatin landscape for B cell programming. Immunity. 2016;44:527-41.

40. Biddie SC, John S, Sabo PJ, Thurman RE, Johnson TA, Schiltz RL, et al. Transcription factor AP1 potentiates chromatin accessibility and glucocorticoid receptor binding. Mol Cell. 2011;43:145-55.

41. Ren G, Cui K, Zhang Z, Zhao K. Division of labor between IRF1 and IRF2 in regulating different stages of transcriptional activation in cellular antiviral activities. Cell Biosci. 2015;5:17.

42. Soufi A, Garcia MF, Jaroszewicz A, Osman N, Pellegrini M, Zaret KS. Pioneer transcription factors target partial DNA motifs on nucleosomes to initiate reprogramming. Cell. 2015;161:555-68.

43. Bock C, Beerman I, Lien WH, Smith ZD, Gu H, Boyle P, et al. DNA methylation dynamics during in vivo differentiation of blood and skin stem cells. Mol Cell. 2012;47:633-47.

44. Cabezas-Wallscheid N, Klimmeck D, Hansson J, Lipka DB, Reyes A, Wang Q et al. Identification of regulatory networks in HSCs and their immediate progeny via integrated proteome, transcriptome, and DNA methylome analysis. Cell Stem Cell. 2014;15:507-22.
45. Lipka DB, Wang Q, Cabezas-Wallscheid N, Klimmeck D, Weichenhan D, Herrmann C, et al. Identification of DNA methylation changes at cis -regulatory elements during early steps of HSC differentiation using tagmentation-based whole genome bisulfite sequencing. Cell Cycle. 2014;13:3476-87.

46. Bichi R, Shinton SA, Martin ES, Koval A, Calin GA, Cesari R, et al. Human chronic lymphocytic leukemia modeled in mouse by targeted TCL1 expression. Proc Natl Acad Sci U S A. 2002;99:6955-60.

47. Raval A, Lucas DM, Matkovic JJ, Bennett KL, Liyanarachchi S, Young DC, et al. TWIST2 demonstrates differential methylation in immunoglobulin variable heavy chain mutated and unmutated chronic lymphocytic leukemia. J Clin Oncol. 2005;23:3877-85.

48. Yuille MR, Condie A, Stone EM, Wilsher J, Bradshaw PS, Brooks L, et al. TCL1 is activated by chromosomal rearrangement or by hypomethylation. Genes Chromosomes Cancer. 2001;30:336-41.

49. Cahill N, Rosenquist R. Uncovering the DNA methylome in chronic lymphocytic leukemia. Epigenetics. 2013;8:138-48.

50. Melki JR, Vincent PC, Brown RD, Clark SJ. Hypermethylation of E-cadherin in leukemia. Blood. 2000;95:3208-13.

51. Bechter OE, Eisterer W, Dlaska M, Kuhr T, Thaler J. CpG island methylation of the hTERT promoter is associated with lower telomerase activity in B-cell lymphocytic leukemia. Exp Hematol. 2002;30:26-33.

52. Chantepie SP, Vaur D, Grunau C, Salaun V, Briand M, Parienti JJ, et al. ZAP-70 intron1 DNA methylation status: determination by pyrosequencing in B chronic lymphocytic leukemia. Leuk Res. 2010;34:800-8.

53. Wiestner A, Rosenwald A, Barry TS, Wright G, Davis RE, Henrickson SE, et al. ZAP-70 expression identifies a chronic lymphocytic leukemia subtype with unmutated immunoglobulin genes, inferior clinical outcome, and distinct gene expression profile. Blood. 2003;101:4944-51.

54. Strathdee G, Sim A, Parker A, Oscier D, Brown R. Promoter hypermethylation silences expression of the HoxA4 gene and correlates with IgVh mutational status in CLL. Leukemia. 2006;20:1326-9.

55. Zenz T. Exhausting T cells in CLL. Blood. 2013;121:1485-6.

56. Hanna BS, Roessner PM, Scheffold A, Jebaraj BMC, Demerdash Y, Ozturk S, et al. PI3Kdelta inhibition modulates regulatory and effector T-cell differentiation and function in chronic lymphocytic leukemia. Leukemia. 2019;33:1427-38.

57. Lewinsky H, Barak AF, Huber V, Kramer MP, Radomir L, Sever L, et al. CD84 regulates PD-1/PD-L1 expression and function in chronic lymphocytic leukemia. J Clin Invest. 2018;128:5465-78.

58. Hanna BS, Roessner PM, Yazdanparast H, Colomer D, Campo E, Kugler S, et al. Control of chronic lymphocytic leukemia development by clonallyexpanded CD8(+) T-cells that undergo functional exhaustion in secondary lymphoid tissues. Leukemia. 2019;33:625-37.

59. Forconi F, Moss P. Perturbation of the normal immune system in patients with CLL. Blood. 2015;126:573-81.

60. Joller N, Kuchroo VK. Tim-3, Lag-3, and TIGIT. Curr Top Microbiol Immunol. 2017:410:127-56.

61. Josefsson SE, Beiske K, Blaker YN, Forsund MS, Holte H, Ostenstad B, et al. TIGIT and PD-1 mark intratumoral T cells with reduced effector function in B-cell non-Hodgkin lymphoma. Cancer Immunol Res. 2019;7:355-62.

62. Catakovic K, Gassner FJ, Ratswohl C, Zaborsky N, Rebhandl S, Schubert M, et al. TIGIT expressing CD4+T cells represent a tumor-supportive T cell subset in chronic lymphocytic leukemia. Oncoimmunology. 2017;7:e1371399.

63. Johnston RJ, Comps-Agrar L, Hackney J, Yu X, Huseni M, Yang Y, et al. The immunoreceptor TIGIT regulates antitumor and antiviral CD8(+) T cell effector function. Cancer Cell. 2014;26:923-37.

64. Deng M, Gui X, Kim J, Xie L, Chen W, Li Z, et al. LILRB4 signalling in leukaemia cells mediates $T$ cell suppression and tumour infiltration. Nature. 2018:562:605-9.

65. John S, Chen H, Deng M, Gui X, Wu G, Chen W, et al. A novel anti-LILRB4 CAR-T cell for the treatment of monocytic AML. Mol Ther. 2018;26:2487-95.

66. Zurli V, Wimmer G, Cattaneo F, Candi V, Cencini E, Gozzetti A, et al. Ectopic ILT3 controls BCR-dependent activation of Akt in B-cell chronic lymphocytic leukemia. Blood. 2017;130:2006-17.

67. Zhang T, Jiang B, Zou ST, Liu F, Hua D. Overexpression of B7-H3 augments anti-apoptosis of colorectal cancer cells by Jak2-STAT3. World J Gastroenterol. 2015;21:1804-13.

68. Ramsay AG, Clear AJ, Fatah R, Gribben JG. Multiple inhibitory ligands induce impaired T-cell immunologic synapse function in chronic lymphocytic leukemia that can be blocked with lenalidomide: establishing a reversible immune evasion mechanism in human cancer. Blood. 2012;120:1412-21. 
69. Inamura K, Takazawa Y, Inoue Y, Yokouchi Y, Kobayashi M, Saiura A, et al Tumor B7-H3 (CD276) expression and survival in pancreatic cancer. J Clin Med. 2018;7(7):E172. https://doi.org/10.3390/jcm7070172. PMID 29996538.

70. Roth TJ, Sheinin Y, Lohse CM, Kuntz SM, Frigola X, Inman BA, et al. B7-H3 ligand expression by prostate cancer: a novel marker of prognosis and potential target for therapy. Cancer Res. 2007:67:7893-900.

71. Wang L, Kang FB, Shan BE. B7-H3-mediated tumor immunology: friend or foe? Int J Cancer. 2014;134:2764-71.

72. Krivtsov AV, Figueroa ME, Sinha AU, Stubbs MC, Feng Z, Valk PJ, et al. Cell of origin determines clinically relevant subtypes of MLL-rearranged AML. Leukemia. 2013;27:852-60.

73. Alcantara Llaguno S, Chen J, Kwon C-H, Jackson EL, Li Y, Burns DK, et al. Malignant astrocytomas originate from neural stem/progenitor cells in a somatic tumor suppressor mouse model. Cancer Cell. 2009;15:45-56.

74. Lai A, Kharbanda S, Pope WB, Tran A, Solis OE, Peale F, et al. Evidence for sequenced molecular evolution of IDH1 mutant glioblastoma from a distinct cell of origin. J Clin Oncol. 2011;29:4482-90.

75. Verhaak RG, Hoadley KA, Purdom E, Wang V, Qi Y, Wilkerson MD, et al. Integrated genomic analysis identifies clinically relevant subtypes of glioblastoma characterized by abnormalities in PDGFRA, IDH1, EGFR, and NF1. Cancer Cell. 2010;17:98-110.

76. Capper D, Jones DTW, Sill M, Hovestadt V, Schrimpf D, Sturm D, et al. DNA methylation-based classification of central nervous system tumours. Nature. 2018;555:469-74.

77. Sturm D, Witt H, Hovestadt V, Khuong-Quang DA, Jones DT, Konermann C, et al. Hotspot mutations in $\mathrm{H} 3 \mathrm{~F} 3 \mathrm{~A}$ and $\mathrm{IDH} 1$ define distinct epigenetic and biological subgroups of glioblastoma. Cancer Cell. 2012;22:425-37.

78. Blanpain C. Tracing the cellular origin of cancer. Nat Cell Biol. 2013;15:126-34.

79. Barker N, Ridgway RA, van Es JH, van de Wetering M, Begthel $H$, van den Born $M$, et al. Crypt stem cells as the cells-of-origin of intestinal cancer. Nature. 2009;457:608-11.

80. Zhu L, Gibson P, Currle DS, Tong Y, Richardson RJ, Bayazitov IT, et al. Prominin 1 marks intestinal stem cells that are susceptible to neoplastic transformation. Nature. 2009;457:603-7.

81. Sangiorgi E, Capecchi MR. Bmi1 is expressed in vivo in intestinal stem cells. Nat Genet. 2008;40:915-20

82. Westphalen CB, Asfaha S, Hayakawa Y, Takemoto Y, Lukin DJ, Nuber AH, et al. Long-lived intestinal tuft cells serve as colon cancer-initiating cells. J Clin Invest. 2014;124:1283-95.

83. Powell AE, Wang Y, Li Y, Poulin EJ, Means AL, Washington MK, et al. The pan-ErbB negative regulator Lrig1 is an intestinal stem cell marker that functions as a tumor suppressor. Cell. 2012;149:146-58.

84. Schwitalla S, Fingerle AA, Cammareri P, Nebelsiek T, Goktuna SI, Ziegler PK, et al. Intestinal tumorigenesis initiated by dedifferentiation and acquisition of stem-cell-like properties. Cell. 2013;152:25-38.

85. Queiros AC, Beekman R, Vilarrasa-Blasi R, Duran-Ferrer M, Clot G, Merkel A, et al. Decoding the DNA methylome of mantle cell lymphoma in the light of the entire B cell lineage. Cancer Cell. 2016;30:806-21.

86. Shaknovich R, Geng H, Johnson NA, Tsikitas L, Cerchietti L, Greally JM, et al. DNA methylation signatures define molecular subtypes of diffuse large Bcell lymphoma. Blood. 2010;116:e81-9.

87. Liu Y, Easton J, Shao Y, Maciaszek J, Wang Z, Wilkinson MR, et al. The genomic landscape of pediatric and young adult T-lineage acute lymphoblastic leukemia. Nat Genet. 2017;49:1211-8.

88. Soulier J, Clappier E, Cayuela JM, Regnault A, Garcia-Peydro M, Dombret H, et al. HOXA genes are included in genetic and biologic networks defining human acute T-cell leukemia (T-ALL). Blood. 2005;106:274-86.

89. Huber W, Carey VJ, Gentleman R, Anders S, Carlson M, Carvalho BS, et al. Orchestrating high-throughput genomic analysis with bioconductor. Nat Methods. 2015;12:115-21.

90. Davis CA, Hitz BC, Sloan CA, Chan ET, Davidson JM, Gabdank I, et al. The Encyclopedia of DNA elements (ENCODE): data portal update. Nucleic Acids Res. 2018:46:D794-801.

91. Hsu S-D, Lin F-M, Wu W-Y, Liang C, Huang W-C, Chan W-L, et al. miRTarBase: a database curates experimentally validated microRNA-target interactions. Nucleic Acids Res. 2011;39:D163-9.

92. Karagkouni D, Paraskevopoulou MD, Chatzopoulos S, Vlachos IS, Tastsoglou S, Kanellos I, et al. DIANA-TarBase v8: a decade-long collection of experimentally supported miRNA-gene interactions. Nucleic Acids Res. 2018:46:D239-45.
93. Betel D, Wilson M, Gabow A, Marks DS, Sander C. The microRNA.org resource: targets and expression. Nucleic Acids Res 2008;36:D149-D153.

94. Griffiths-Jones S. miRBase: microRNA sequences, targets and gene nomenclature. Nucleic Acids Res. 2006;34:D140-4

\section{Publisher's Note}

Springer Nature remains neutral with regard to jurisdictional claims in published maps and institutional affiliations.
Ready to submit your research? Choose BMC and benefit from:

- fast, convenient online submission

- thorough peer review by experienced researchers in your field

- rapid publication on acceptance

- support for research data, including large and complex data types

- gold Open Access which fosters wider collaboration and increased citations

- maximum visibility for your research: over $100 \mathrm{M}$ website views per year

At BMC, research is always in progress.

Learn more biomedcentral.com/submissions 